\title{
Intensity Measures of Consumer Preference
}

\author{
JOHN R. HAUSER \\ Northwestern University, Evanston, Illinois \\ STEVEN M. SHUGAN \\ University of Chicago, Chicago, Illinois \\ (Received August 1978; accepted September 1979)
}

\begin{abstract}
To design successful new products and services, managers need to measure consumer preferences relative to product attributes. Many existing methods use ordinal measures. Intensity measures have the potential to provide more information per question, thus allowing more accurate models or fewer consumer questions (lower survey cost, less consumer wearout). To exploit this potential, researchers must be able to identify how consumers react to these questions and must be able to estimate intensity-based preference functions. This paper provides a general structure for using intensity measures for estimating consumer preference functions. Within the structure: (1) alternative measurement theories are reviewed, (2) axioms for developing testable implications of each theory are provided, (3) statistical tests to test these implications and distinguish which theory describes how consumers are using the intensity measures are developed, (4) functional forms appropriate for the preference functions implied by each theory are derived, and (5) procedures to estimate the parameters of these preference functions are provided. Based on these results, a practical procedure, implemented by an interactive computer package, to measure preference functions in a market research environment is developed. An empirical case illustrates how the statistical tests and estimation procedures are used to aid in the design of new telecommunications devices. Empirical results suggest the majority of consumers can provide intensity judgments. The intensity-based estimation procedures do better on several criteria than ordinal estimation.
\end{abstract}

$\mathrm{T}$ O DESIGN and evaluate new product or service strategies, managers require an understanding of how consumers form preferences and how they will behave if a new product or service is launched. Accurate predictions on consumer response coupled with models of production costs, tax rates, cash flow, and product-line considerations can lead to more successful products and can reduce the risk of failure [46].

Many researchers have investigated the twin problems of understanding consumer preference and predicting consumer choice. Some techniques estimate consumer preference functions by representing "consumer utility" as a function of the product's attribute levels $[13,14,18$, $24,29,41,49]$. (These techniques assume either (1) the product with the 
largest "utility" is chosen, or (2) the higher the "utility" of a product, the more likely a consumer is to choose that product.) For a review of this literature, see Green and Srinivasan [13]. Such techniques are useful in the product design because they indicate the relative effects of changes in the attributes of that product. Other techniques measure interval or ratio-scaled preference directly based on actual products prior to test market or national introduction. For example, see Silk and Urban [48]. These techniques are useful in the evaluation of new products because they are based on actual products and on strong direct preference measures.

Conjoint analysis (Luce and Tukey [34], Tversky [53], Green and Srinivasan [13]) is one effective technique to measure preference functions. Conjoint analysis has been quite successful in marketing (Green and Devita [11], Green and Wind [14], Wind and Spitz [57]), but the application of conjoint analysis can be improved. The consumer task can be quite tedious, often requiring each consumer to rank order 20-40 "products" in terms of preference. (Products can be real or represented by attribute levels.) The number of products is usually kept at a minimum with a fractional factorial design $[6,14]$. Furthermore, the measurement estimates ordinal preference, i.e., a ranking over products, rather than intensity of preference, i.e., how much a product is preferred over another. Finally, because the conjoint measurement task can be tedious, it is difficult to ask further questions to check behavioral assumptions underlying the preference measurement. It is possible to use a form of conjoint analysis called tradeoff analysis (Johnson $[24,25])$ which reduces the consumer task by having consumers rank order products where only two attributes vary at a time. Preference measures are still ordinal and assumptions still difficult to check.

If conjoint analysis or tradeoff analysis were extended by the use of intensity measures for preference, it would be possible to gather more information per question. With the proper theoretical structure and estimation technique, this detailed information could be used to develop preference models providing a more accurate description of the consumer evaluation process. Also, if estimation requires fewer consumer measurements, more questions can be asked to test behavioral assumptions and to help understand the evaluation process.

One form of preference intensity measures is von Neumann-Morgenstern utility theory [55]. Preference is ordinal; however, the preference function can handle products with uncertain attributes. The consumer task, indifference measurement, provides more information per question and the axiomatic theory allows the measurement of more complex preference functions $[17,18]$. Some underlying behavioral assumptions can be checked (preferential indifference, utility independence, see Keeney $[26,28])$, but it remains infeasible to check all behavioral assumptions 
as is done in the lengthy interviews (often 2 days or more) of prescriptive applications (Keeney [27], Keeney and Raiffa [29], Farquhar [8]). Even with indifference questions and axiomatic theory, marketing applications still require a 40-50-minute personal interview to measure a consumer's preference function. Furthermore, the theory cannot yet handle the measurement error inherent in consumer interviews.

Another form of preference intensity measures is constant sum paired comparisons (CSPC). With CSPC, consumers are asked to allocate fixed sum of points or "chips" between pairs of actual products or product concepts in proportion to their preferences for those products. Ratioscaled preference scales are developed from analyses of these responses (Torgerson [52]). Silk and Urban [48] report ease of measurement and excellent predictive capability in over 10 product categories. As high as $80 \%$ of the uncertainty is explained where uncertainty is measured by information theory (Hauser [15]). (Unfortunately, pure constant sum measurement, in which chips are allocated simultaneously among all products, is a difficult consumer task often leading to inaccurate results, Pessemier [42].) But the applications to data have not developed preference functions. Instead, they have developed composite measures, i.e., a preference value for each product. To design new products and to better understand the consumer response, we need preference functions which identify how consumers use product attributes to form preferences.

Intensity measures ask respondents for more information about their preferences. Therefore, CSPC measures provide the potential to estimate more accurate preference functions and to do so using fewer consumer questions. All we need to do is test the data to find out if it does, indeed, contain the additional information. Unfortunately, the axioms of conjoint and utility theories are based on ordinal measurement. Consequently, any estimation based on these axioms would only use the ordinal properties of the CSPC measures and neglect the intensity information inherent in the measures. Furthermore, as we will show, there are many alternative hypotheses regarding exactly what the intensity portion of the CSPC measurement means to the consumer. To use CSPC measure for preference functions, we must develop tests to identify how consumers interpret the CSPC task and we must develop estimation procedures incorporating the intensity information.

A third form of preference intensity measures is graded paired comparisons (GPC). With GPC, consumers are asked to choose between two stimuli and to indicate the intensity of their preference between the stimuli. In marketing, Huber and Sheluga [21] and Neslin [40] have used GPC measures to estimate consumer preference functions and/or functions mapping product attributes to perceptions. Both applications report good empirical results using analysis of variance estimation based on an assumption of interval intensity information. 
But interval is only one possible assumption. Other assumptions about how consumers allocate chips may lead to improved preference functions with different scale properties. If we can develop a common structure which incorporates the interval assumption as well as the ordinal (conjoint, utility) assumption and possibly other assumptions, and if we develop statistical tests to distinguish among these assumptions, we can improve the GPC applications and extend the CSPC task to measure and estimate preference functions.

This paper provides a general structure for using intensity measures for estimating consumer preference functions. Within the structure we (1) review alternative measurement theories, (2) provide axioms for developing testable implications of each theory, (3) develop statistical tests to test these implications and distinguish which theory describes how consumers are using the intensity measures, (4) derive functional forms appropriate for the preference functions implied by each theory, and (5) provide procedures to estimate the parameters of these preference functions.

\section{INTENSITY MEASURES AND THEORY}

To develop and evaluate new products and marketing strategies, we must develop preference functions relating the attributes of potential products to consumer preference for those products. The goal of the measurement and estimation is to understand and to predict a consumer's preferences by observing his(her) perceptions of each product in a choice set relative to a set of attributes (e.g., quality, personalness, convenience, and value for health services). The preference function $(c: X \rightarrow C)$ maps the attribute perceptions $(X)$ into a scalar measure of preference $(C)$, such that the consumer is most likely to prefer the product with the largest scalar measure. (We do not require perfect prediction due to measurement variance, incomplete specification and other random errors.) The remainder of this paper will assume that any feasible product can be represented by a set of attributes, $X=X_{1} \bullet X_{2} \bullet \cdots \cdot X_{K}$. Let $x_{j k}$ be the level of attribute $k$ for product $j$ and let $x_{j}=\left\{x_{j 1}, x_{j 2}, \cdots, x_{j K}\right\}$. Various methods to identify and measure these attributes are factor analysis (Urban [54]), discriminant analysis (Johnson [23], Pessemier [42]), and similarity scaling (Green and Rao [12]). Alternatively, one can use physical characteristics (e.g., lens speed and exposure time for cameras) in place of measured perceptions.

An important objective in the following discussion is the development of preference functions rather than composite preference values. Thus, we will be concerned with attribute-based measurement rather than estimating preference values for existing products. Nonetheless, each of the theories or models and statistical tests apply to the special case of 
composite measures. In this case there is only one attribute, product identity. For a discussion of composite measures versus preference functions (component measures) with GPC, see Huber and Sheluga [21].

All analyses and measurements are presented at the level of the individual consumer, however, the theory can be readily modified for aggregate level analysis. With individual measures demand is estimated by aggregating predicted individual preference behavior or by using models that translate preference to choice. The measurement is attributebased. We illustrate the theory with CSPC measurement, but indicate extensions to GPC measures and other intensity measures such as dollar metric (Pessemier [42]). For example, Figure 1 schematically represents one form of CSPC measurement. The consumer is given two product concepts (or occasionally existing products) with known or measured attribute levels, $\underline{x}_{1}$ and $\underline{x}_{2}$, and asked to allocate a fixed sum $(S)$ of "chips"

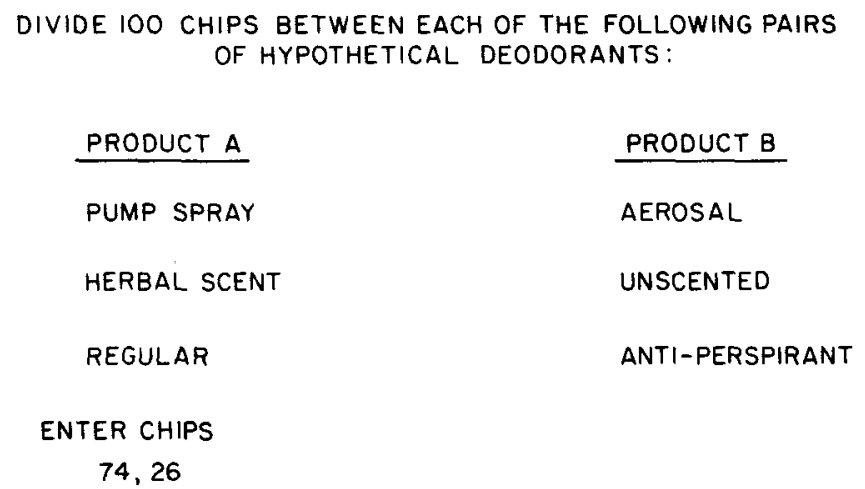

PRODUCT A

PUMP SPRAY

HERBAL SCENT

REGULAR

ENTER CHIPS

74,26

PRODUCT B

AEROSAL

UNSCENTED

ANTI-PERSPIRANT

Figure 1. An example of constant sum paired comparison measurement.

between the products according to his(her) preferences for those products.

Suppose the consumer allocates $a_{12}$ chips to $\underline{x}_{1}$ and $a_{21}=S-a_{12}$ chips to $x_{2}$. If the mechanism for allocation is left unspecified as in Figure 1, there exists some ambiguity as to what type of preference the pair $\left(a_{12}\right.$, $a_{21}$ ) represents. Perhaps $a_{12}>a_{21}$ means only that $x_{1}$ is preferred to $x_{2}$. In this case the CSPC measure is no better than simple pairwise comparisons (Johnson [25]) and one would continue to use conjoint analysis, tradeoff analysis, or von Neumann-Morgenstern theory.

But perhaps more information is contained in the measurement (i.e., chip allocation). One possible assumption is based on a cardinal utility theory developed by Shapley [44]. Here the allocation is made so that $c\left(x_{1}\right)-c\left(x_{2}\right)$ and $a_{12}-a_{21}$ are proportional. This assumption implicitly implies $c(\cdot)$ has at least interval scale properties and chips are allocated 
by differences. Another stronger assumption, following Torgerson's ratioscaled postulates [50], is that $c\left(x_{1}\right) / c\left(x_{2}\right)=a_{12} / a_{21}$. This assumption implies $c(\cdot)$ has at least ratio scale properties and chips are allocated by ratios. By making full use of the measured preferences, either of these assumptions yields preference functions which measure intensity of preference, but each theory requires (a) a test for the appropriateness of the assumptions [i.e. how chips are allocated by differences, ratios, etc.] and (b) a method to estimate the preference function, $c\left(x_{j}\right)$.

If the researcher has some indication of how the consumer will respond to the CSPC measurement, he(she) may assume one theory, say ratio, and encourage the consumer to respond according to that theory. For example, additional instructions may request the consumer to "allocate chips in proportion to the ratio of your preferences for the hypothetical products." In this case the general structure and statistical tests can be used for theory testing rather than for theory identification. If the tests do not reject the theory, the same estimation procedures apply.

We begin with a general structure. This structure provides a common notation that clarifies the parallel assumptions of alternative theories and allows each theory to be formulated as a special case of a general axiom system. We will formulate alternative theories by adding behavioral assumptions which specialize the structure and which adapt it to applications. Statistical tests determine whether the data are consistent with the behavioral assumptions (e.g., merely random, ordinal, interval or ratio).

\section{General Structure}

Let $c(\cdot)$ be a preference function mapping attribute levels into a realvalue depicting preference. Suppose that a particular consumer is presented with two potential products, $i$ and $j$, with known attribute levels, $\underline{x}_{i}$ and $\underline{x}_{j}$, and suppose he(she) responds to the CSPC question with the pair $\left(a_{i j}, a_{j i}\right)$ where $a_{i j}+a_{j i}=S$. For example, in Figure $1, x_{i}$ would represent the attribute levels for product $A$ and $\left(a_{i j}, a_{j i}\right)$ would equal (74, 26). We implicitly define the properties of the preference function for this consumer by the equation:

$$
c\left(x_{i}\right) * c\left(\underline{x}_{j}\right)=a_{i j} \circ a_{j i}
$$

where the measurement relation, $\circ$, indicates mathematically how the consumer reacts to the CSPC measures thus specifying how consumers allocate chips. The property operator, *, indicates the corresponding mathematical relationship among the preference values thus specifying minimal scale properties for $c(\cdot)$. (For GPC and other intensity measures, replace $a_{i j}{ }^{\circ} a_{j i}$ by $f\left(g_{i j}\right)$ where $g_{i j}$ is the graded scale comparing $i$ and $j$ and $f(\cdot)$ is a function dependent upon the theory represented by ${ }^{\circ}$.) 
To be practical, $c(\cdot)$ will be measured for a relatively small subset of the possible elements of $X$. To be useful, $c(\cdot)$ must apply to all elements of $X$. This requires consistency in the form of symmetry, identity, and property transitivity. We formally express these properties with 3 axioms. Later, we develop statistical tests to empirically test special cases of this general axiom system. The first property is symmetry. Symmetry simply states "the order of evaluation does not affect $c(\cdot)$." (We assume any instrument bias such as order effects have been statistically corrected or randomized.)

Aхıом 1 (Symmetry). If $c\left(\underline{x}_{i}\right) * c\left(\underline{x}_{j}\right)=a_{i j}^{\circ} a_{j i}$ then $c\left(\underline{x}_{j}\right) * c\left(\underline{x}_{i}\right)=a_{j i} \circ a_{i j}$.

Identity states that once instrument bias is corrected, an equal allocation among the stimuli means the consumer is indifferent among the stimuli. Mathematically, identity is a necessary property of the measurement relation.

Axiom 2 (Identity). $a_{i j}{ }^{\circ} a_{i j}=I_{0}$ for all $a_{i j}$ where $a_{i j} \circ I_{0}=a_{i j}$.

Symmetry and identity are binary relations; property transitivity is a test for consistency among three or more stimuli. Property transitivity will provide a method for testing the validity of a particular measurement relationship. Given three responses, Axiom 3 defines the necessary relationship among the responses, thus creating a statistically testable implication. We must first define a transitive operator, $T$, that acts to reverse the measurement relation. The third component of Definition 1 is necessary to ensure that $T$ is not a null operator.

DEFINITION 1 (Transitivity Operator). $T$ is a transitivity operator for the measurement relation, ${ }^{\circ}$, if $T$ is commutative, $\left(a_{i j}{ }^{\circ} a_{j i}\right) T\left(a_{j i}{ }^{\circ} a_{i j}\right)=I_{0}$, and $\left(a_{i j}^{\circ} a_{j i}\right) T a_{j i}=a_{i j}$.

Axıом 3 (Transitivity). If $T$ is a transitivity operator associated with $\circ$, then $\left(a_{i j} \circ a_{j i}\right) T\left(a_{j k} \circ a_{k j}\right) T\left(a_{k i} \circ a_{i k}\right)=I_{0}$. When $T$ is not associative, Axiom 3 holds if at least one of the possible groupings holds.

Finally, we do not want our analyses to be logically dependent upon the choice of the constant sum, $S$. Thus, subject to measurement and roundoff errors, we require Axioms 1, 2, and 3 to hold for any $S$.

To simplify measurement, we investigate whether $c(\cdot)$ can be separated into uni-attributed functions. For example, $c\left(\underline{x}_{i}\right)$ would be simpler to estimate if it were separable into a sum of $K$ uni-attributed functions, $c_{1}\left(x_{1 j}\right), c_{2}\left(x_{2 j}\right)$, etc. The separable form depends on $*$ and $\circ$, but in each case there are identifiable independence properties which imply the form of separability. These independence properties are special cases of evaluative independence, defined as follows.

Suppose that $X=X_{1} \bullet X_{2} \bullet \cdots \cdot X_{K}$ are partitioned into $Y=X_{1} \bullet X_{2} \bullet$ $\cdots \cdot X_{m}$ and $Z=X_{m+1} \bullet X_{m+2} \bullet \cdots \cdot X_{K}$. Let $\underline{y}_{i}, \underline{y}_{j} \in Y$, let $\underline{z}_{i}, \underline{z}_{j} \in Z$, then 
$\underline{x}_{i}=\left(\underline{y}_{i}, \underline{z}_{i}\right)$ etc. As a special case, define $X_{\bar{k}}=X_{1} \bullet X_{2} \mathbf{X}_{k-1} \bullet X_{k+1} \cdot \ldots \cdot X_{K}$. Define $X_{k j}$ similarly. We can define a general independence property, evaluative independence. We later use this property to specify functional forms for the consumer utility function. If evaluative independence holds for a given theory, then we show that specific functional forms apply for the consumer's utility function and, hence, different estimation procedures are appropriate.

Definition 2. Let $Y, Z$ be a partition of $X$. Then for a given consumer, $Y$ is evaluative independent of $Z$ (written $Y$ e.i. Z). If for any $z \circ \in Z$

$$
c\left(\underline{y}_{i}, \underline{z}^{\circ}\right) * c\left(\underline{y}_{j}, \underline{z}^{\circ}\right)=a_{i j} \circ a_{j i}
$$

then

$$
c\left(\underline{y}_{i}, z\right) * c\left(\underline{y}_{j}, z\right)=a_{i j} \circ a_{j i} \text { for all } z \in Z .
$$

In words, the consumer's answers depend only upon the attributes, $Y$, varying among the products in the pair. If evaluative independence does not hold, the theories may still apply, but separability cannot be exploited in the estimation procedures. Evaluative independence is a generalization of independence properties found in utility theory (Keeney and Raiffa [29]).

To proceed further, we must make assumptions with respect to *,, and the implied $T$. We now proceed to develop statistical tests capable of rejecting the axiom system for particular operators.

\section{Notation}

In the following discussion, we use standard matrix notation. Let $M: R \times C$ denote a matrix with $R$ rows and $C$ columns. Let $M^{\prime}$ denote the transposed matrix. Let $r(M)$ equal rank of $M$ and let $M^{-1}$ denote the inverse of $M$ if its exists, otherwise let $M^{-1}$ denote the pseudo inverse of $M$ (Caradus [5]). Let $\underline{I}$ denote the identity matrix, $\underline{1}$ a matrix of only ones, and $\underline{0}$ is a matrix of only zeros. Finally, if dimensions are not specified, assume they are conformable.

\section{Interval Theory}

Interval theory, one special case of our axiom system, assumes that the consumer allocates the chips such that the difference, $d_{i j}=a_{i j}-a_{j i}$, indicates the intensity of preference. Shapley [44] derives fundamental axioms implying the existence of an interval preference function. Within the general structure, interval theory is given by (2).

$$
c\left(x_{i}\right)-c\left(\underline{x}_{j}\right)=a_{i j}-a_{j i} .
$$

The property operator and measurement relation are both subtraction, the transitivity property operator is addition, and the identity element is 
zero. Then Axiom 3 for interval theory requires (3) to hold for all triplets of product concepts, $\underline{x}_{i}, \underline{x}_{j}$, and $\underline{x}_{k}$. (Test Axioms 1 and 2 in an analogous manner.)

$$
\left(a_{i j}-a_{j i}\right)+\left(a_{j k}-a_{k j}\right)+\left(a_{k i}-a_{i k}\right)=0 .
$$

In practice, measurement error may prevent (3) from holding precisely. Hence, the left-hand side of (3) may be some (not necessarily zero) number $\nu_{i j k}^{I}$. The superscript $I$ denotes interval theory. Let $\nu_{t}^{I}=\left(\nu_{i j k}^{I}\right)$ for transitivity test $t$. A useful measure of fit is then sum of squared interval error, $E^{I}$, shown in (4).

$$
E^{I}=\sum_{t=1}^{m}\left(\nu_{t}^{I}\right)^{2}=\nu^{P} \nu^{I}
$$

where $t$ indexes the transitivity tests (specific triplets), $v^{I}$ is the vector of $\nu_{t}^{I}$ 's, and $m$ is the number of such tests. $E^{I}$ is a useful intuitive measure: but to test the theory, we must investigate distributional properties of $E^{I}$ and related statistics. Then if $E^{I}$ is significantly small (statistically), we say interval theory is consistent with the data.

Assume that there exists an interval measurement error $\epsilon_{i j}^{Y}$ distributed normally with mean $u_{I}$ and variance $\sigma_{I}^{2}$ [denoted $\left.N\left(u_{I}, \sigma_{I}^{2}\right)\right]$. (We do not need the assumption of zero-mean.) Then the observed value of $a_{i j}$ is given by:

$$
a_{i j}=a_{i j}^{I}+\epsilon_{i j}^{I}
$$

where $a_{i j}^{I}$ is the true chip allocation under the interval assumption. Make similar assumptions for $a_{j i}, a_{j k}, a_{k j}, a_{i k}$, and $a_{k i}$. To derive the distribution for $E^{I}$, we must consider the relationship of the errors for the terms in 3 .

Assumption 1. In a CSPC measurement, the consumer first selects $a_{i j}$ and sets $a_{j i}=S-a_{i j}$.

Under Assumption 1, $a_{j i}$ is functionally related to $a_{i j}$; thus if error is uncorrelated across measurements, $\nu^{I}$ is the sum of three independent normal random variables each with variance $s_{I}^{2}=4 \sigma_{I}^{2}$. Under the hypotheses of interval theory, (3) holds and the expected value of $v^{I}$ is zero. Thus $\nu^{I}$ is distributed as $N\left(0,12 \sigma^{2}\right)$.

AssumPTION 2. In a CSPC measurement, the consumer first selects $d_{i j}$ $=a_{i j}-a_{j i}$ then adjusts both $\alpha_{i j}$ and $a_{j i}$ such that $a_{i j}+a_{j i}=S$.

Under Assumption 2 the errors $\epsilon_{i j}^{I}$ and $\epsilon_{j i}^{I}$ are not perfectly correlated. If they are uncorrelated, $\nu^{I}$ is the sum of six independent normal random variables and $\nu^{I}$ is distributed as $N\left(0,6 \sigma_{I}^{2}\right)$. In general, there will be some negative correlation $\rho$ and the variance of $E^{I}$ will be given by $6 \sigma_{I}{ }^{2}-6 \rho_{I} \sigma_{I}{ }^{2}$ where $-1 \leq \rho_{I} \leq 0$ (Mood and Greybill [38], Theorems 9 and 10). Assumption 1 implies $\rho_{I} \equiv-1$. Assumption 2 (extreme case) implies $\rho_{I}$ $\equiv 0$. In general $v^{I}$ is zero-mean normal random variable with variance $s_{I}^{2}$ 
$<6\left(1-\rho_{I}\right) \sigma_{I}^{2}$. Note that whether or not the variables are independent and regardless of the value of $s_{I}^{2}, \nu^{I}$ will be normally distributed (with mean zero for interval theory).

If the transitivity tests were independent, then $E^{I}$ would be the sum of squared independent normal variates and would be a chi-squared $\left(\chi^{2}\right)$ variate. Unfortunately, not all transitivity tests will be independent, because the same $\epsilon_{i j}^{I}$ can appear in more than one transitivity test.

Let $\nu^{I}: m \times 1$ be the vector of $m$ transitivity tests. Let $d^{I}: D \times 1$ be the data vector of differences (we will suppress the $I$ for notational simplicity), i.e., $d^{I}=\left[a_{i j}-a_{j i}, a_{r s}-a_{s r}, \cdots\right]^{\prime}$. Let $M: m \times D$ be the experimental design matrix identifying transitivity tests. Then, $\nu=M d$. For example,

$$
\nu=\left[\nu_{1} \nu_{2}\right]^{\prime}=\left[\begin{array}{ccccc}
1 & 1 & 1 & 0 & 0 \\
0 & 0 & 1 & 1 & 1
\end{array}\right]\left[d_{i j} d_{j k} d_{k i} d_{i q} d_{q k}\right]^{\prime}
$$

$M$ should be formulated such that the transitivity tests are not redundant, i.e. such that $r(M)=m$. Otherwise the experimentor may assume more degrees of freedom than allowable by the experimental design. The maximum rank for $M$ is the number of different products in the design minus two. From the preceding arguments, $d$ has a nonsingular multivariate ( $D$-variate) normal distribution with mean $\mu: D \times 1$ and covariance matrix $\Sigma: D \times D$, denoted $d \sim N_{D}(\mu, \Sigma)$. Therefore, if $r(M)=m$, then (Giri, [10, Theorem 4.1.5])

$$
\nu \sim N_{m}\left(M \mu, M \Sigma M^{\prime}\right)
$$

and if $A=\left(M \Sigma M^{\prime}\right)^{-1}$ is the inverse (or generalized inverse, Caradus [5]), of $M \Sigma M^{\prime}$, then ([10, Theorem 6.2.2]) $\nu^{\prime} A v$ has a noncentral $\chi^{2}$ distribution with $m$ degrees of freedom and noncentrality parameter $n_{1}=\mu^{\prime} M^{\prime} A M \mu$. We will call $\nu^{\prime} A \nu$ the adjusted sum of squared error, denoted $\mathscr{E}^{\prime}$. Now for interval theory, $M \mu=\underline{0}$. Hence,

THEOREM 1. For interval theory, the adjusted sum of squared error, $\mathscr{E}^{I}$ $=\nu^{\prime} A \nu$ is $\chi^{2}$ distributed with $m$ degrees of freedom where $\nu=M d, A=$ $\left(M \Sigma M^{\prime}\right)^{-1}$ and $r(M)=m$.

Here $\mathscr{E}^{I}$ represents the weighted sum of squared errors across transitivity tests. The weights are determined by the measurement covariance modified by the experimental design. Note that $\mathscr{E} I$ simplifies for the special case when (1) the design is orthogonal $\left(M M^{\prime}=I\right)$, and (2) the errors are homoscedastic and independent for successive pairs, i.e., $\Sigma=s^{2} I$. Under these conditions, $\mathscr{E}^{d}$ becomes $E^{I} / s^{2}$.

We see $\mathscr{E}^{I}$ provides a test statistic for interval theory when $\Sigma$ or $s^{2}$ is known. That is, given confidence level $\alpha$, reject interval theory if $\mathscr{E}^{I}>K_{\alpha}$ where $K_{\alpha}$ is the value of a cumulative $\chi^{2}$ distribution with $m$ degrees of freedom at probability level $1-\alpha$.

However, in general $\Sigma$ is unknown. In that case assume independence 
and homoscedasticity across measurements, that is, $\Sigma=s^{2} \underline{I}$. Alternatively, we could assume some other special structure for $\Sigma$. Note that these assumptions do not prevent the measurement means from being functionally related. Now, let

$$
\eta=W d \text { where } W: w \times D \text { satisfies } M W^{\prime}=\underline{0} .
$$

Now, $\nu$ and $\eta$ are independent (Giri [10, Theorem 6.2.3]) because $M W^{\prime}$ $=\underline{0}$. The vector $\nu$ can be interpreted as the weighted sum of arbitrarily chosen chip allocations. It is, therefore, logical to choose $W$ such that each row has exactly three nonzero elements, each being \pm 1 . Together, $M$ and the maximum rank $W$ represent all orthogonal triplets. $M$ represents the independent triplets implied by Axiom 3 and $W$ represents other orthogonal triplets. If $B$ is the inverse or generalized inverse of $s^{2} W W^{\prime}$, then $\eta^{\prime} B \eta$ is a noncentral $\chi^{2}$ variate [43] with $w=r(W)$ degrees of freedom and noncentrality parameter $n_{2}=E[\eta]^{\prime} W^{\prime} B W E[\eta]$ where $E[\cdot]$ denotes expected value. If $A=\left(M \Sigma M^{\prime}\right)^{-1}$, then

$$
\begin{aligned}
(m / w)\left(\eta^{\prime} B \eta\right) /\left(\nu^{\prime} A \nu\right)=(m / w)\left(s^{-2} \eta^{\prime}\right. & \left.\left(W W^{\prime}\right)^{-1} \eta\right) /\left(s^{-2} \nu^{\prime}\left(M M^{\prime}\right)^{-1} v\right) \\
& =\left(\eta^{\prime}\left(W W^{\prime}\right)^{-1} \eta\right) /\left(\nu^{\prime}\left(M M^{\prime}\right)^{-1} \nu\right)(m / w)
\end{aligned}
$$

has no unknown parameters. It has a doubly noncentral $F$-distribution (see Giri $[10, p .109]$ ) with $m, w$ degrees of freedom and noncentrality parameters $n_{1}$ and $n_{2}$. As a null hypothesis, we assume interval theory is not correct. Under this hypothesis, $E\left[\nu^{\prime} A v / m\right]=E\left[\eta^{\prime} B \eta / w\right]$. Intuitively, the null says the variance left unexplained by our theory is of the same magnitude or greater than the general variability that is not covered by the theory (correct by degrees of freedom). Under the null hypothesis $n_{1}$ $=n_{2}$ equals some $n$. Theorem 2 defines $Q^{I}$ which is the ratio of general variance over the variance left unexplained by interval theory.

TheOREM 2. For the null hypothesis, if $W$ is defined such that $M W^{\prime}=$ 0 and if errors are independent and homoscedastic across measurements, then

$$
Q^{I}=\left(\eta^{\prime}\left(W W^{\prime}\right)^{-1} \eta / w\right) /\left(\nu^{\prime}\left(M M^{\prime}\right)^{-1} \nu / m\right)
$$

has a doubly noncentral $F$-distribution with $(w, m)$ degrees of freedom, with equal noncentrality parameters.

If more than one $W$ exists such that $M W^{\prime}=\underline{0}$, then we take that $W$ such that $w=r(W)$, the number of degrees of freedom, is maximized (tending to a more powerful test). Then interval theory is supported when $Q^{I}$ in Theorem 2 is greater than $K_{\alpha}(n)$ where $K_{\alpha}(n)$ is determined from a noncentral $F$-distribution with noncentrality parameters both equal to $n$. Note, the ratio can be interpreted as the inverse of the percent unexplained variance in the transitivity tests. The ratio $\eta^{\prime}\left(W W^{\prime}\right)^{-1} \eta /$ $\left[\nu^{\prime}(M M)^{-1} \nu+\eta^{\prime}(W W)^{-1} \eta\right]$ is the percent explained variance. Hence, as 
the variability explained by interval theory increase, $Q^{l}$ increases. If transitivity tests are perfectly satisfied without error, $\nu=\underline{0}$ and $Q^{I}$ approaches infinity.

Next we must find the noncentrality parameter $n$. Rejection of the null hypothesis supports interval theory, therefore $n$ is chosen to bias the test toward accepting the null hypothesis (making the test more conservative). Theorem $2^{\prime}$ provides a method for doing this.

THEOREM 2 . If $K_{\alpha}(n)$ is the cutoff value for rejecting the null hypothesis given noncentrality parameter $n$ and confidence level $\alpha, K_{\alpha}(0)$ is the cutoff value for probability $1-\alpha$ for a cumulative $F$-distribution and $K_{\alpha}(0)>1$, then $K_{\alpha}(n) \leq K_{\alpha}(0)$.

The conservative test for interval theory becomes,

Reject the hypothesis that interval transitivity does not explain a significant amount of triplet variability at confidence level $\alpha$ if and only if $Q^{I} \geq K_{\alpha}(0)$ where $K_{\alpha}(0)$ is determined from a central cumulative $F$ distribution with $w, m$ degrees of freedom.

Hence $Q^{I} \geq K_{\alpha}(0)$ supports interval theory. Note that this is a very conservative test for interval theory (i.e., tending not to support interval theory). Intuitively, setting $n=0$ assumes arbitrarily chosen differences add to zero, thereby exaggerating measurement variance and flattening the $\chi^{2}$ distribution. In effect, the test acts as if $\alpha$ were smaller than the one specified. Finally, increasing the number of transitivity tests tends to more powerful tests.

There are three final comments. First, in practice the $W$ matrix is unique with $w<m$. It can be found by exhaustively enumerating all linearly independent vectors to the $M$ matrix. Second, if we wish to test a sample of people to find if the entire sample is interval (rather than each individual), then let $\eta=\nu-\bar{\nu}$ where $\vec{\nu}$ is the sample mean. The ratio of Theorem 2 has a central $F$-distribution under the interval theory hypothesis. Third, Theorems 1, 2, and $2^{\prime}$ can also be proven for GPC measures. Simply define $g$ as the vector of signed intensity judgments, e.g., $g_{i j}, g_{r q}, \cdots$, and replace $d$ by $g$ in the above theorems. For the independent, homoscedastic case, $\Sigma=s^{2} I$.

Theorems 1,2 , and $2^{\prime}$ provide a useful battery of tests. If $\rho$ and $\sigma^{2}$, or more simply $s^{2}$, are known from external experiments, the $\chi^{2}$ test provides a statistical test of the axioms. Alternatively, Theorem 1 can be used to jointly test interval theory and an error variance. If the error variance is unknown, Theorem 2 , or more simply Theorem $2^{\prime}$, still provides a test of interval theory, but because $s^{2}$ is free to vary, Theorem $2^{\prime}$ will not be as stringent a test as Theorem 1.

If interval theory is not rejected, we proceed to estimate $c\left(x_{i}\right)$. Many decompositions are possible. We show one particularly useful decomposition based on each attribute being evaluative independent of its com- 
plement set. Based on this simple proof, one can readily extrapolate the ideas to less restrictive functional forms when certain interactions are important.

Theorem 3. For the interval theory, $X_{k}$ e.i. $X_{\bar{k}}$ for all $k$ implies $c\left(\underline{x}_{j}\right)=$ $c_{1}\left(x_{1 j}\right)+c_{2}\left(x_{2 j}\right)+\cdots+c_{K}\left(x_{K j}\right)$.

Proof. $Y$ e.i. $Z$ implies by Definition 1 that $c\left(y_{i}, z^{\circ}\right)-c\left(y_{j}, z^{\circ}\right)=c\left(y_{i}\right.$, $\underline{z})-c\left(\underline{y}_{j}, z\right)$ for all $z$, thus $c\left(\underline{y}_{i}, \underline{z}\right)-c\left(\underline{y}_{j}, z\right)=f\left(\underline{y}_{i}, y_{j}\right)$. Assume that $c(\cdot, \cdot)$ is differentiable. Then $\partial f\left(\underline{y}_{i}, \underline{y}_{j}\right) / \partial z=0$. Thus $\partial c\left(\underline{y}_{i}, \underline{z}\right) / \partial z=$ $\partial c\left(\underline{y}_{j}, \underline{z}\right) / \partial z$ for all $\underline{y}_{i}, \underline{y}_{j}$. Thus $\partial c(\underline{y}, z) / \partial z=\gamma^{\prime}(z)$. Integrating gives $c(y, z)=\gamma(z)+\beta(y)$. Thus, for $Y \equiv X_{1}, c\left(x_{j 1}, x_{j 1}\right)=c_{i}\left(x_{j 1}\right)+c_{1}\left(x_{j 1}\right)$. Continuing we get $c\left(x_{j 1}, x_{j 2}, x_{j 12}\right)=c_{1}\left(x_{j 1}\right)+c_{2}\left(x_{j 2}\right)+c_{12}\left(x_{j 12}\right)$, etc., until the result is attained. The proof when $c(\cdot, \cdot)$ is not differentiable is similar but more tedious. (Note that $Y$ e.i. $Z$ is reflexive for the interval theory, i.e. $Y$ e.i. $Z$ implies $Z$ e.i. $Y$ ). Furthermore, this theorem could easily have been proven with telescoping sets such as $X_{k}$ e.i. $X_{k+1} \bullet X_{k+2} \bullet$ $\ldots X_{K}$.

If Theorem 2 holds, then $c\left(x_{j}\right)$ can be estimated with linear programs based on an absolute error structure or with ordinary least squares regression (OLS). Simply discretize each attribute and define:

$$
c\left(\underline{x}_{j}\right)=\Sigma_{k} \Sigma_{l} \lambda_{l k} \delta_{m k j}
$$

where $\lambda_{l k}=$ the utility derived from attribute $k$ at level $l$.

$\delta_{l k j}=1$ if $x_{k j}$ is at the $l$ th level and $\delta_{l k j}=0$ otherwise. The estimation equation is:

$$
a_{i j}-a_{j i}=\Sigma_{k} \Sigma_{l} \lambda_{l k}\left(\delta_{l k i}-\delta_{l k j}\right)+\text { error. }
$$

In the linear programming formulation, (7) is used to define absolute error which is then minimized subject to any constraints on the $\lambda_{l k}$. This algorithm is a modification of LINMAP (Srinivasan and Shocker [49]) for intensity measures. Empirically, we have found that the linear program performs better on fit and predictability measures than OLS because one can easily add prior information (known by the researcher) to the constraints in the linear program. For example, if the $\lambda_{l k}$ are known to be monotonic in $l$, a monotonic constraint can be added to the estimation:

$$
\lambda_{l, k}<\lambda_{l+1, k} \quad i=1, \cdots, L-1 \quad(L=\text { no. of levels }) .
$$

Or if an ideal level, $\hat{l}$, is specified,

$$
\lambda_{l, k}<\lambda_{l+1, k}, \quad i=1, \cdots, \hat{l}-1 \quad \text { and } \lambda_{l, k}>\lambda_{l+1, k}, \quad i=\hat{l}, \cdots, L-1 .
$$

If there are $L$ levels, a minimum of $(L-1) K$ questions must be asked to specify $c(\cdot)$. (Base points for $c_{k}(\cdot)$ are chosen such that no redundancy exists.) More questions are required when an error term is included in (7) 
and estimates, $\hat{\lambda}_{l k}$, are obtained. Theorem 3 and (7) apply to GPC measures with $g_{i j}=a_{i j}-a_{j i}$. For an illustrative example of the interval theory analysis see Appendix 3.

\section{Ratio Theory}

Ratio theory is another special case of our axiom system. It assumes that the consumer allocates chips such that the ratio $a_{i j} / a_{j i}$, indicates the intensity of preference. This theory was originally formulated by Torgerson [52] to produce composite preference measures rather than preference functions. (In Torgerson's measurement, consumers are explicitly requested to use the CSPC scale as a ratio scale.) Within the general structure, ratio theory is given by:

$$
c\left(\underline{x}_{i}\right) / c\left(\underline{x}_{j}\right)=a_{i j} / a_{j i}
$$

The transitivity operator is multiplication, the identity element is 1.0, and Axiom 3 becomes

$$
\left(a_{i j} / a_{j i}\right) \cdot\left(a_{j k} / a_{k j}\right) \cdot\left(a_{i k} / a_{k i}\right)=1 .
$$

Note that (8) does not necessarily require a zero-point for $c\left(x_{i}\right)$ within the range of feasible products. In (8) we are concerned primarily with the method in which consumers allocate chips as well as with the scale properties of the preference scale. It is possible that preference is a ratio scale, but (8) does not apply because the consumer reacts differently to the CSPC measurement. Similar comments apply to (2) in the interval theory.

Axiom 3 for ratio theory can be tested by checking (9), which must hold for all product triplets. (Test Axioms 1 and 2 in an analogous manner.) As in interval theory, we expect measurement error. In general, the left-hand side of (9) will equal some nonunity number, $v_{i j k}^{R}$ (where $R$ superscript denotes ratio theory). For convenience we define $v_{t}^{R}=\log \left(v_{t}^{R}\right)$ for transitivity test $t$. A useful measure of fit is the sum-of-squared ratio error, $E^{R}$, given by:

$$
E^{R}=\sum_{t=1}^{m}\left(\nu_{t}^{R}\right)^{2}=\nu^{R^{\prime}} \nu^{R}
$$

where $\nu^{R}$ is the vector of $\nu_{t}^{R}$ 's.

Again, for ratio theory we assume the error in $\nu^{R}$ is normally distributed. Therefore, $v^{R}$ has a lognormal error. If we assume that the observed value of $a_{i j}$ is given by:

$$
a_{i j}=a_{i j}^{R} \cdot \epsilon_{i j}^{R}
$$

where $\epsilon_{i j}^{R}$ is $\Lambda\left(u_{R}, \sigma_{R}{ }^{2}\right)$ and $a_{i j}^{R}$ is the true chip allocation under the ratio assumption. $\left[\Lambda\left(u_{R}, \sigma_{R}^{2}\right)\right.$ is a lognormal random variable with generating parameters $u_{R}$ and $\sigma_{R}{ }^{2}$. That is, $\log \Lambda\left(u_{R}, \sigma_{R}{ }^{2}\right)$ is $N\left(u_{R}, \sigma_{R}{ }^{2}\right)$.] Make similar assumptions for $a_{j i}, a_{j k}, a_{k j}, a_{i k}$ and $a_{k i}$. 
Using the reproductive properties of the lognormal distribution (Aitchison and Brown [2, Theorem 2.3]), one can show that the hypotheses of ratio theory imply that $\nu^{R}$ is a zero-mean normal random variable with variance $s_{R}{ }^{2}=6\left(1-\rho_{R}\right) \sigma_{R}{ }^{2}$ where $\rho_{R}$ is the correlation between $\log \epsilon_{i j}^{R}$ and $\log \epsilon_{j i}^{R}$, and $\sigma_{R}^{2}$ is the variance of $\left(\log \epsilon_{i j}^{R}\right)$.

Following the development for interval theory, we define $\nu^{R}: m \times 1$ as the vector of transitivity errors. Let $d^{R}: D \times 1$ be the vector of log-ratios, i.e., $d^{R}=\left[\log \left(a_{i j} / a_{j i}\right), \log \left(a_{r s} / a_{s r}\right), \cdots\right]$. (The $R$ superscripts denoting ratio theory on $\nu^{R}$ and $d^{R}$ are suppressed in the subsequent development when there is no possibility of confusion with $\nu^{I}$ and $d^{I}$ from interval theory.) Then $\nu=M d$ where $M$ is the same experimental design matrix defined earlier. Using the properties of the lognormal distribution, $d$ has a multivariate normal distribution with mean $\mu: D \times 1$ and covariance matrix $\Sigma: D \times D$ and $\nu \sim N_{m}\left(M \mu, M \Sigma M^{\prime}\right)$. When the errors across measurements are independent and homoscedastic, $\Sigma=s^{2} I$. If $M^{\prime} M=I$ then $\nu^{\prime}\left(M \Sigma M^{\prime}\right)^{-1} \nu=E^{2} / s^{2}$. Theorems 4 and 5 provide the test for ratio theory. For GPC, replace $\log \left(a_{i j} / a_{j i}\right)$ by a signed value of $\log \left(g_{i j}\right)$. The sign indicates whether $i$ is preferred to $j$ or vice versa.

THEOREM 4. For ratio theory, $\nu^{\prime}\left(M \Sigma M^{\prime}\right)^{-1} \nu$ is $\chi^{2}$ distributed with $m$ degrees of freedom where $\nu=M d$.

THEOREM 5. For ratio theory, if $W$ is defined such that $M W^{\prime}=0$ and if errors are independent and homoscedastic across measurements, then $Q^{R}=\left(\eta^{\prime}\left(M W^{\prime}\right)^{-1} \eta / w\right) /\left(\nu^{\prime}\left(M M^{\prime-1} \nu / m\right)\right.$ where $\eta=W d$ has a doubly noncentral $F$-distribution with $(w, m)$ degrees of freedom, and equal noncentrality parameters.

Using Theorem 2', the conservative test for ratio theory at confidence level 2 becomes,

Reject a random chip allocation for ratio theory if and only if $Q^{R} \geq$ $K_{\alpha}(0)$ where $K_{\alpha}(0)$ is determined from a central cumulative $F$-distribution with $w, m$ degrees of freedom.

Finally, in some cases, we will use Theorems 1 and 4 to compare interval and ratio theories. While it is reasonable to assume $\rho_{I} \cong \rho_{R}$, in general, $\sigma_{R} \neq \sigma_{I}$. To provide equivalent tests for the ratio and interval theories, we must relate $\sigma_{R}$ to $\sigma_{I}$. For equivalence, we require that the variance of $a_{i j}$ due to measurement error be the same for both theories. Using the lognormal distribution and (11), we can show that the variance of $a_{i j}$ due to $\epsilon_{i j}^{R}$ is $\left(E\left[a_{i j}^{R}\right]\right)^{2}\left[\exp \left(\sigma_{R}^{2}\right)-1\right]$ where $E\left[a_{i j}^{R}\right]$ is the expected value of $a_{i j}^{R}$ (Aitchison and Brown [2, p. 8]). The equal variance requirement then gives:

$$
\sigma_{R}^{2}=\log \left[\sigma_{I}^{2} /\left(E\left[\alpha_{i j}^{R}\right]\right)^{2}+1\right]
$$

If we know or wish to test an error variance, $\sigma_{I}{ }^{2}$, then we match $\sigma_{R}{ }^{2}$ and use Theorem 4. If $\sigma_{R}{ }^{2}$ is unknown, use Theorem 5. 
If ratio theory is not rejected, we proceed to estimate $c\left(x_{j}\right)$. As in interval theory, we can use evaluative independence to derive decompositions for $c\left(x_{j}\right)$. For example, the following theorem parallels Theorem 1 in the interval theory. Again, Theorem 6 can be extended as needed.

Theorem 6. For the ratio theory, $X_{k}$ e.i. $X_{\bar{k}}$ for all $k$ implies $c\left(\underline{x}_{j}\right)=$ $c_{1}\left(x_{i j}\right) \cdot c_{2}\left(x_{2 j}\right) \cdot \cdots \cdot c_{K}\left(x_{K j}\right)$.

Proof. $Y$ e.i. $Z$ implies by definition 1 that $c\left(\underline{y}_{i}, z^{\circ}\right) / c\left(\underline{y}_{j}, z^{\circ}\right)=c\left(\underline{y}_{i}\right.$, $z) / c\left(\underline{y_{j}}, z\right)$ for all $z$, thus $c\left(\underline{y}_{i}, \underline{z}\right) / c\left(\underline{y}_{j}, z\right)=h\left(\underline{y}_{i}, \underline{y}_{j}\right)$. Thus, $\log c\left(\underline{y}_{i}, z\right)$ $-\log c\left(\underline{y}_{j}, z\right)=\log h\left(\underline{y}_{i}, \underline{y}_{j}\right)=f\left(\underline{y}_{i}, \underline{y}_{j}\right)$. Following the proof to Theorem 1 then gives $\log c\left(\underline{x}_{j}\right)=\log c\left(x_{j 1}\right)+\log c_{2}\left(x_{j 2}\right)+\cdots+\log c_{K}\left(x_{j K}\right)$ which is the result.

If Theorem 6 holds, the $c\left(x_{j}\right)$ can be estimated by taking logarithms of (8) and formulating a linear program for absolute error or by using OLS. Simply discretize each attribute and define:

$$
c\left(\underline{x}_{j}\right)=\Pi_{k} \Pi_{l}\left(\lambda_{l k}\right)^{\delta_{k j}}
$$

where $\delta_{l k j}$ is defined as before. To estimate $\lambda_{l k}$ use the equation:

$$
\log \left(a_{i j} / a_{j i}\right)=\Sigma_{k} \Sigma_{l}\left(\delta_{l k i}-\delta_{l k j}\right) \log \lambda_{l k}+\text { error. }
$$

For $L$ levels, $(L-1) \cdot K$ questions are required to specify $c(\cdot)$, more if the $\log \lambda_{l k}$ 's are to be estimated. Theorem 6 and (14) apply to GPC measures with $a_{i j} / a_{j i}=g_{i j}$ when $i$ is preferred to $j$ and $a_{i j} / a_{j i}=1 / g_{i j}$ when $j$ is preferred to $i$.

\section{Hybrid Model}

If ratio theory is rejected and interval theory is not rejected, we use the interval theory. If interval theory is rejected but not ratio, we use ratio theory. But it is possible that the tests reject neither theory. In this case, we can use either theory or, heuristically, select the theory with the best fit to the data.

Another alternative, that is suggested by the general structure, is to formulate a hybrid model by carefully specifying the relationship between $c\left(x_{i}\right)$ and $a_{i j}$. We use the word model since axioms, distributional assumptions, and tests would be required for a theory. In this case, our axiom system would have to be extended to a more general system. One hybrid model that combines the features of interval and ratio theories is given by:

$$
c\left(\underline{x}_{i}\right)-\left(a_{i j} / a_{j i}\right)^{\gamma} c\left(\underline{x}_{j}\right)=\beta\left(a_{i j}-a_{j i}\right)
$$

When $\gamma \rightarrow 0, \beta \rightarrow 1$ the interval theory applies; when $\gamma \rightarrow 1, \beta \rightarrow 0$ the ratio theory applies. While a hybrid theory based on (15) is complex, the 
hybrid model can be estimated by nonlinear estimation procedures such as OPTISEP [1] and SUMT [7].

In the special case when $\gamma=0$, then (15) is a simple extension of (2). Similarly, if $\beta=0,(15)$ is an extension of (8). In either case, the estimation equation is linear in the new parameter and linear programming or OLS can be used.

\section{Ordinal Theory (Utility Theory, Conjoint Analysis)}

If both the ratio theory and the interval theory are rejected, then we test the hypothesis that the CSPC questions measure ordinal preference. That is, $a_{i j}>a_{j i}$ means that $x_{i}$ is preferred to $x_{j}$. This is the case of either von Neumann-Morgenstern utility theory or more simply ordinal utility theory. See von Neumann and Morgenstern [55], Friedman and Savage [9], Marschak [36], Herstein and Milner [19], and Jensen [22] for axioms which imply the existence of such functions. If the data structure has reasonable properties ('Tversky [53], Krantz et al. [30], and Luce and Tukey [34]) and sufficient measurements are made, conjoint analysis can be used.

To put ordinal theory in the general structure, define an indicator function, $\delta(t)$, such that $\delta(t)=-1$ if $t<0, \delta(t)=0$ if $t=0$, and $\delta(t)=1$ if $t>0$.

$$
\delta\left[c\left(\underline{x}_{i}\right)-c\left(\underline{x}_{j}\right)\right]=\delta\left(a_{i j}-a_{j i}\right) .
$$

A test for Axiom 3 is ordinary transitivity. (Note that both ordinal properties and the stronger intensity properties can hold simultaneously.) If $\delta_{i j}=\delta\left(a_{i j}-a_{j i}\right)$, then the test for ordinal transitivity can be written as:

$$
\delta_{i j} T \delta_{j k} T \delta_{k i}=0
$$

where

$$
\delta_{p q} T \delta_{r s}=\delta\left[\delta_{p q}+\delta_{r s}\right]
$$

Here $T$ is not associative and hence all cases must be checked. This is equivalent to testing whether the following equation holds (the proof of equivalence is by exhaustive enumeration):

$$
\delta_{i j}+\delta_{j k}+\delta_{k i}+\delta_{i j} \delta_{j k} \delta_{k i}=0
$$

which covers all possible preference and indifference orderings among $x_{i}$, $\underline{x}_{j}$, and $\underline{x}_{k}$.

Following the intensity theories, let the left-hand side of equation 18 be $\nu^{0}$ where the superscript 0 denotes ordinal theory. Since we are concerned with the ordinal properties, we use $\delta^{2}\left(v^{0}\right)$, which acts as an indicator function for errors in (18). A useful measure of fit is the number of times, $E^{0}$, that ordinal properties do not hold where $E^{0}=\sum_{t=1}^{m} \delta^{2}\left(\nu_{t}^{0}\right)$.

If the consumer gives consistent ordinal preferences, then we expect (18) to hold more often than if the consumer allocates $\delta_{i j}$ randomly. Our test is formulated to reject random allocation. One random assumption 
is that the three values of $\delta_{i j},(-1,0,1)$, are equally likely. Another assumption is that only strong orders occur, $\delta_{i j}=-1$ or 1 , and they are equally likely. In general, we assume that indifference, $\delta_{i j}=0$, occurs with probability $q$ and the other values of $\delta_{i j}$ each occur with probability ( 1 $-q) / 2$. By exhaustive enumeration, one can show that, under the random assumption, $\nu_{0} \neq 0$ with probability, $p$, given by:

$$
p=2[(1-q) / 2]^{3}+6 q[(1-q) / 2]^{2}+6 q^{2}[(1-q) / 2] .
$$

For example, if $q$ is zero, then $p=(1 / 4)$; if $q=(1 / 3)$, then $p=(14 / 27)$. These are bounds on $p$ for $q \in[0,1 / 3]$. In general, $1 / 4 \leq p \leq 14 / 27$. The first value, $p$ $=1 / 4$ assumes all preference orderings are equally likely. If we assume chips are allocated completely randomly, then $\alpha_{i j}$ is uniformly distributed and $q=1 /(S+1)$. Then when $S$ grows large, the first value $(p=1 / 4)$ is approached.

If the errors in the transitivity tests are independent, $E^{0}$ can be thought of as resulting from $m$ independent Bernoulli trials with success probability given by $p$. This is stated formally in the following theorem.

TheOREM 7. Let $p$ be given by (19). Then the cumulative distribution of $E^{0}$ is given by $\operatorname{Prob}\left[E^{0} \leq E\right]=\sum_{k=0}^{E}\left(\begin{array}{c}m \\ k\end{array}\right) p^{k}(1-p)^{m-k}$.

As in interval and ratio theory, the errors in the transitivity tests in an arbitrary experimental design matrix are not independent, in which case the distribution for $E^{0}$ can be derived analytically for any given $M$ as the exact distribution of interdependent Bernoulli trials; but it is notationally and computationally cumbersome and must be done for each $M$. Fortunately, simulation results show Theorem 7 to be a good approximation for the $M$ matrix used in our empirical work (see Appendix 1).

Theorem 7 sets confidence levels for rejecting the random hypothesis. For example, if $m=8$, then $E^{0} \leq 1$ rejects, at the 0.05 level, the hypothesis that $(-1,0,1)$ are equally likely for $\delta_{i j}$. Under the same hypothesis, $E^{0}$ $\leq 2$ rejects at the 0.15 level. Under the hypothesis of only strong orders $E^{0}=0$ corresponds to the 0.10 level and $E^{0} \leq 1$ corresponds to the 0.37 level. The same tests apply for any ordinal measurement or for the ordinal properties of GPC and other intensity measures.

If the random hypothesis is rejected, we can use utility theory (Hauser and Urban [18]) or conjoint analysis (Green and Srinivasan [13]) to estimate the preference function, $c\left(x_{i}\right)$. Once again, evaluative independence yields decompositions that simplify estimation. Evaluative independence becomes preferential independence (Keeney and Raiffa [29]) and $Y$ e.i. $Z$ implies that there exists a value function $v(y)$ such that $c\left(x_{j}\right)$ $=u\left[v\left(\underline{y}_{j}\right), \underline{z}_{j}\right]$. In particular, if each pair of attributes is preferential independent of the other attributes, then there exists some ordinal $c(\cdot)$ which decomposes additively separable. This is stated formally in Theo- 
rem 8. The proof is contained in Ting [51], Farquhar [8], or Keeney and Raiffa [29].

Theorem 8. For ordinal theory, $X_{k} \cdot X_{j}$ e.i. $X_{\bar{k}}$ for all $k$ implies there is some $c(\cdot)$ such that $c\left(x_{j}\right)=c_{1}\left(x_{1 j}\right)+c_{2}\left(x_{2 j}\right)+\cdots+c_{K}\left(x_{K j}\right)$.

If the consumer task involves stimuli with uncertain attributes, i.e., lotteries, then the axiom structure for $c\left(x_{i}\right)$ implies $c\left(x_{i}\right)$ is unique to a positive linear transformation (von Neumann and Morgenstern [55]) and evaluative independence becomes utility independence and mutual utility independence implies the quasi-additive form (Keeney [26]). Theorem 9 formalizes these implications.

Theorem 9. For von Neumann-Morganstern utility theory, $X_{k}$ e.i. $X_{\bar{k}}$ for all $k$ implies $c\left(x_{j}\right)=\sum_{k} \lambda_{k} c_{k}\left(x_{k j}\right)+\sum_{m>k} \sum_{k} \lambda_{m k} c_{m}\left(x_{m j}\right) c_{k}\left(x_{k j}\right)+$ third order terms $+\cdots+$ Kth order terms where $\lambda_{k}, \lambda_{m k}$, etc. are scalar constants.

For estimation procedures applicable to ordinal theory, see [11, 13, 14, $24,30,41,49,53]$; for von Neumann-Morgenstern theory, see $[8,16,18$, $29,30]$.

\section{Stochastic Theory I}

If $E_{0}$ rejects ordinal theory in favor of a random allocation, then we must investigate theories more general than deterministic preference. We could interpret $a_{i j}>a_{j i}$ as meaning the probability of choosing $x_{i}$ is greater than the probability of choosing $x_{j}$. For axioms implying the existence of $c\left(x_{i}\right)$ under this interpretation, see Hauser [16]. For conditions leading to probabilistic choice, see Shugan [45]. There exists many interpretations of stochastic theory. We now derive one CSPC theory consistent with probabilistic preference. The theory, again, concerns chip allocation and the implied estimation, rather than, the consumer's mental processes.

Let $P\left[x_{i}>x_{j}\right]$ be the probability that the consumer will prefer $x_{i}$ to $x_{j}$ in a paired comparison. Now suppose (1) chips are allocated sequentially to achieve the chip allocation $\left(a_{i j}, a_{j i}\right)$. For each toss, i.e. individual "chip" allocation, there is a probability $P_{m i j}$ that the $m$ th "chip" will be allocated to product $i$ rather than to product $j$. If one assumes (2) a stationary process (i.e., $P_{i j}=P_{m i j}=P_{n i j}$ for all $n, m$ ); (3) $P_{i j}=P\left[x_{i}>x_{j}\right]$, and since $P_{i j}+P_{j i}=1$, then given Axiom 3, each individual chip allocation is Bernoulli. For details see Appendix 2. The total chip allocation for any fixed sum of "chips" is given by:

$$
\operatorname{Prob}\left[a_{i j}, a_{j i}\right]=\left(\begin{array}{c}
a_{i j}+a_{j i} \\
a_{i j}
\end{array}\right)\left[P_{i j}\right]^{a_{i j}\left[P_{j i}\right]^{a_{j i}}}
$$

The maximum-likelihood estimator for $P\left(x_{i}>x_{j}\right]$ for a single comparison 
is given by:

$$
P_{i j}=P\left[\underline{x}_{i}>\underline{x}_{j}\right]=a_{i j} /\left(a_{i j}+a_{j i}\right)
$$

If $P\left(x_{i}>x_{j}\right)$ is a function, $f(\cdot, \cdot)$, of only the preference values, then (21) becomes a special case of the general structure. That is:

$$
f\left[c\left(x_{i}\right), c\left(\underline{x}_{j}\right)\right]=a_{i j} /\left(a_{i j}+a_{j i}\right)
$$

A special case of stochastic theory is the logit model [37] where

$$
f\left[c\left(\underline{x}_{i}\right), c\left(\underline{x}_{j}\right)\right]=\exp \left[c\left(\underline{x}_{i}\right)\right] /\left(\exp \left[c\left(\underline{x}_{i}\right)\right]+\exp \left[c\left(\underline{x}_{j}\right)\right]\right)
$$

In this case, Axiom 3 (property transitivity) becomes Luce's choice axiom $[32,33]$ :

$$
\left(P_{i j} / P_{j i}\right) \cdot\left(P_{j k} / P_{k j}\right) \cdot\left(P_{k i} / P_{i k}\right)=1 .
$$

For sufficient conditions for Luce's axiom and a survey of its extensions, see Shugan [45]. To test stochastic Theory I, we formulate a likelihood ratio test on the basis of (20). Since estimation is analytically cumbersome, we refer the reader to Appendix 2. (It is interesting to note if $S$ is sufficiently large and if neither $a_{i j}$ nor $a_{j i}$ is close to zero, then (20) becomes approximately normal (Mood and Greybill, [38, Theorem 7.5]) which is consistent with an assumption of normally distributed measurement error.)

(23) can be estimated with standard maximum likelihood-based computer packages (Berkman et al. [4]). Evaluative independence implies a multiplicative decomposition of $\exp \left[c\left(x_{i}\right)\right]$ which indicates an additive decomposition of $c\left(x_{i}\right)$. (22) can be extended with an exponent for $a_{i j}$ and $a_{j i}$ (Stevens [50], Pessemier [42]) and (23) can be extended to probit and other probabilistic models. To use stochastic Theory I for GPC, one must assume that $g_{i j}$ is a probability scale.

\section{Stochastic Theory II}

If the ratio, interval, ordinal, and stochastic theories are all rejected, we must search for alternative explanations. One such explanation is provided by Bass [3] who derives a theory of stochastic preference that makes no assumptions with respect to specific individual consumers but is based on distributional assumptions about how the market as a whole behaves. (Preference probabilities vary across the consumer population with some known distribution.) For a review article on these models, see Horsky et al. [20].

\section{Summary}

The general structure, $c\left(x_{i}\right) * c\left(x_{j}\right)=a_{j i} \circ a_{j i}$, provides a common link between the measurement theories and models. Within this structure one 
can proceed systematically, searching and testing for the measurement theory that best explains how consumers react to the CSPC scale or other intensity scales. When the proper theory is identified, we posit (and will support with an empirical case) that prediction and understanding is improved.

In exploratory research we suggest the CSPC, GPC, or other intensity tasks be left unspecified and the general structure be used to identify the natural and easiest consumer response to the questions. Then modify the questions to encourage response according to the identified theory and use the statistical tests with more stringent confidence levels to test the theory.

One such search/test/estimation procedure is suggested in Table I. Ratio and interval theories are tested first. If one is clearly identified, it

TABLE I

One Possible Use of the Theory Tests Within the General Structure

\begin{tabular}{|c|c|c|c|c|c|}
\hline \multirow{2}{*}{$\begin{array}{c}\text { Interval } \\
\text { Test } \\
\left(E_{I}\right)\end{array}$} & \multicolumn{2}{|c|}{ Ratio Test $\left(E_{R}\right)$} & \multirow{2}{*}{$\begin{array}{c}\text { Ordinal } \\
\text { Test } \\
\left(E_{0}\right) \\
\end{array}$} & \multicolumn{2}{|c|}{ Likelihood Ratio Test } \\
\hline & Accept & Reject & & Accept & Reject \\
\hline Accept & $\begin{array}{c}\text { Best fit or } \\
\text { hybrid } \\
\text { model }\end{array}$ & $\begin{array}{l}\text { Interval } \\
\text { theory }\end{array}$ & Accept & $\begin{array}{l}\text { Ordinal or } \\
\text { stochastic I }\end{array}$ & $\begin{array}{l}\text { Ordinal } \\
\text { theory }\end{array}$ \\
\hline Reject & $\begin{array}{l}\text { Ratio } \\
\text { theory }\end{array}$ & $\begin{array}{l}\text { Further } \\
\text { testing }\end{array}$ & Reject & $\begin{array}{c}\text { Stochastic } \\
\text { theory I }\end{array}$ & $\begin{array}{c}\text { Stochastic } \\
\text { theory II }\end{array}$ \\
\hline
\end{tabular}

is used. If both are accepted, either the best fitting theory or the hybrid model is used. If both are rejected, the ordinal theory is tested and, if accepted, it is used. If the ordinal theory is rejected, stochastic Theory I is tested. If it is rejected, stochastic Theory II is used.

Estimation proceeds only after identification of the appropriate theory. If evaluative independence holds, decomposition simplifies estimation. If evaluative independence is strongly rejected, interaction terms may be required. For brevity we have not derived statistical tests for evaluative independence, although such tests are straightforward employing the assumed distributional properties. Furthermore, even if evaluative independence does not hold, the decompositions may provide good approximations.

The search/test/estimation procedure can proceed at either the individual or the aggregate levels. At the individual level, the best theory is chosen for each consumer in the sample. At the aggregate level, a best representative theory is chosen for the sample. In either case, estimation is at the individual level. In the former case, accuracy is enhanced, but interpersonal comparisons cause difficulties. In the latter case, some accuracy is sacrificed, but the ability to compute summary statistics (mean, median, interquartile interval) can lead to enhanced managerial understanding. 
We now provide a numerical example to illustrate the application of the general structure to CSPC measurement. Section 3 then provides an empirical case from a marketing research application.

\section{NUMERICAL EXAMPLE AND IDENTIFICATION TEST}

Table II is one example of a hypothetical consumer's response to 16 CSPC questions. This design for three attributes at three levels contains 10 tests of Axiom 2 (property transitivity) of which 8 are nonredundant. These are used to select the appropriate theory. For example, question triplets $\{1,3,6\},\{1,4,5\}$, and $\{3,2,4\}$ form three such tests. Further,

TABLE II

One Hypothetical Consumer's Response to a $3 \times 3$ Design Atroibute Levels ${ }^{a}$

\begin{tabular}{|c|c|c|c|c|c|c|c|c|}
\hline \multirow{2}{*}{ Question } & \multicolumn{3}{|c|}{ Product $i\left(x_{i}\right)$} & \multicolumn{3}{|c|}{ Product $j\left(x_{j}\right)$} & \multicolumn{2}{|c|}{ CSPC } \\
\hline & $x_{1}$ & $x_{2}$ & $x_{3}$ & $x_{1}$ & $x_{2}$ & $x_{3}$ & $a_{i j}$ & $a_{j i}$ \\
\hline 1 & $\mathbf{H}$ & $\mathrm{H}$ & $\mathbf{L}$ & $\mathrm{H}$ & L & $\mathrm{H}$ & 43 & 57 \\
\hline 2 & $\mathbf{M}$ & $\mathbf{M}$ & $\mathbf{M}$ & $\mathbf{L}$ & $\mathrm{H}$ & $\mathbf{H}$ & 27 & 73 \\
\hline 3 & $\mathrm{H}$ & $\mathrm{L}$ & $\mathrm{H}$ & $\mathbf{L}$ & $\mathrm{H}$ & $\mathrm{H}$ & 25 & 75 \\
\hline 4 & $\mathbf{M}$ & $\mathbf{M}$ & $\mathbf{M}$ & $\mathbf{H}$ & $\mathrm{L}$ & $\mathrm{H}$ & 53 & 47 \\
\hline 5 & $\mathrm{H}$ & $\mathbf{H}$ & $\mathrm{L}$ & $\mathbf{M}$ & $\mathbf{M}$ & $\mathbf{M}$ & 40 & 60 \\
\hline 6 & $\mathrm{~L}$ & $\mathbf{H}$ & $\mathbf{H}$ & $\mathbf{H}$ & $\mathrm{H}$ & $\mathrm{L}$ & 80 & 20 \\
\hline 7 & L & $\mathbf{H}$ & $\mathrm{L}$ & $\mathbf{L}$ & $\mathrm{L}$ & $\mathrm{H}$ & 43 & 57 \\
\hline 8 & $\mathrm{H}$ & $\mathbf{L}$ & $\mathrm{L}$ & $\mathrm{L}$ & $\mathrm{H}$ & $\mathrm{L}$ & 25 & 75 \\
\hline 9 & $\mathbf{L}$ & $\mathbf{L}$ & $\mathrm{H}$ & $\mathrm{H}$ & $\mathrm{L}$ & L & 80 & 20 \\
\hline 10 & $\mathbf{M}$ & M & $\mathrm{L}$ & $\mathrm{L}$ & $\mathrm{H}$ & $\mathrm{L}$ & 47 & 53 \\
\hline 11 & $\mathrm{~L}$ & $\mathrm{~L}$ & $\mathrm{H}$ & $\mathbf{M}$ & $\mathbf{M}$ & $\mathrm{L}$ & 60 & 40 \\
\hline 12 & $\mathbf{L}$ & $\mathbf{M}$ & $\mathbf{M}$ & $\mathbf{M}$ & $\mathbf{M}$ & $\mathbf{L}$ & 71 & 29 \\
\hline 13 & $\mathbf{H}$ & $\mathrm{L}$ & $\mathrm{L}$ & $\mathrm{L}$ & $\mathbf{M}$ & $\mathbf{M}$ & 13 & 87 \\
\hline 14 & $\mathbf{M}$ & $\mathbf{M}$ & $\mathrm{L}$ & $\mathrm{H}$ & $\mathrm{L}$ & $\mathrm{L}$ & 73 & 27 \\
\hline 15 & $\mathbf{L}$ & $\mathbf{L}$ & $\mathbf{H}$ & $\mathbf{M}$ & $\mathbf{L}$ & $\mathbf{M}$ & 45 & 55 \\
\hline 16 & $\mathbf{L}$ & $\mathrm{H}$ & $\mathrm{L}$ & $\mathbf{M}$ & $\mathrm{L}$ & $\mathbf{M}$ & 47 & 53 \\
\hline
\end{tabular}

${ }^{a} \mathrm{H}, \mathrm{M}, \mathrm{L}=$ high, medium, and low levels of each attribute.

question pairs $\{1,7\},\{3,8\}$, and $\{6,9\}$ each test evaluative independence for one attribute.

This design, one of many, is chosen to illustrate how to test theories and properties. Products (combinations of attribute levels) are orderbalanced to minimize order-bias effects in measurement and approximately balanced across questions. That is, subject to tradeoffs in achieving theory tests, each product appears approximately equally often as $\underline{x}_{i}$ and $\underline{x}_{j}$ and equally often throughout the measurement. (Order balance is not exact because some products appear an odd number of times.) For a discussion of balance, see Huber and Sheluga [21]; for a discussion of experimental designs, see Cochran and Cox [6].

We have chosen $S=100$ chips because empirically the round-off error inherent in a 10-chip allocation makes it difficult to discriminate between 
interval and ratio theories. Furthermore, the normal or lognormal distributions are more reasonable if $S$ is large. However, an optimal value for $S$ could be determined for any particular theory by relating $S$ to the confidence level of the test. Consumer reaction to date has been favorable for an allocation of 100 chips.

\section{Transitivity Tests}

Table II was generated based on a ratio-scaled preference function with a random error of $\sigma=1$ and $\rho=0$ (which is equivalent to $\sigma_{R}=0.02$ for $E\left[\alpha_{i j}^{R}\right]=50$ ). The statistical tests should uncover this fact. First, $E^{0}$ $=0.0$ is significant at the 0.005 level. Thus, the data are at least ordinal. The transitivity experimental design is not orthogonal $\left(M M^{\prime} \neq I\right)$, thus we compute the adjusted mean square error which gives $\mathscr{E}^{I}=33.5$. This value is above the 0.05 level cutoff at 15.5 , thus rejecting the interval theory. The equivalent statistic, $\mathscr{E}^{R}$, equals 0.45 for the ratio theory. This is well below the 0.05 cutoff. Together, the test statistics give evidence in favor of the ratio theory.

Had we not known $\sigma$ and $\rho$, we could have used the $F$-statistic with $K_{0.05}(0)=4.1$. For Table II, $Q^{R}=217.8$ and $Q^{I}=117.1$, indicating that both ratio and interval theories are better explanations of the data than the alternative hypothesis. (Note that ratio theory has a larger statistic.) We compare these statistics to $Q^{R}=1.0$ and $Q^{I}=1.4$ which were obtained from random data. Thus, we see that the $\chi^{2}$ tests are more stringent and should be used if $\sigma$ and $\rho$ are known, but even if they are unknown, the $F$-tests provide good discrimination between intensity theories and random data.

The reader may select $\sigma$ by comparing the CSPC process to a process where the chip allocations are generated from $S$ independent Bernoulli trials. In that case, the error variance is $S a_{i j}\left(1-a_{i j}\right)$. For example, with $S=100$, then $a_{i j}=50$ corresponds to $\sigma=5, a_{i j}=10$ or 90 corresponds to $\sigma=3$, and $a_{i j}=1$ or 99 corresponds to $\sigma=1$. If the true $a_{i j}$ 's are uniformly distributed, then the expected error variance corresponds to $\sigma=4.08$. Interestingly, $\sigma / \sigma_{R}$ is approximately 50 for $\sigma=1,2,3,4$, or 5 . Alternatively, we can use external estimates of $\sigma^{2}$ measured through repetition of the consumer task or we can use post hoc estimates obtained from the error variance in the regressions (7) and (14) used to estimate $c\left(x_{i}\right)$. Future empirical research may use these techniques to establish guidelines for $\sigma^{2}$. The error correlation, $\rho$, is related to the assumptions about how consumers allocate chips. Thus, qualitative discussions with consumers can give guidance to select $\rho$.

\section{Estimation}

After the transitivity tests, a ratio theory preference function was estimated with a linear program minimizing the absolute error in (14). 
The utility values, $\lambda_{l k}$ 's, were constrained to be monotonically increasing in $l$. The estimated parameters, shown in Table IIIa, compare well to the "actual" parameters which produced the data, shown in Table IIIb.

\section{EMPIRICAL EXAMPLE: TELECOMMUNICATIONS}

The previous sections have developed procedures for the identification and estimation of preference functions to measure intensity of preference. This section illustrates these procedures with an empirical example. The results show that at the 0.05 level, $61 \%$ of the tested consumers react to the CSPC measure via a ratio or an interval theory and $83 \%$ are at least ordinal. Furthermore, preference models based on these theories improve

TABLE III

Preference Parameters for the Hypothetical Consumer Responses in TABLE II

\begin{tabular}{lllr}
\hline & $x_{1}$ & $x_{2}$ & \multicolumn{1}{c}{$x_{3}$} \\
\hline H & 2.9 & 8.7 & 11.6 \\
M & 2.0 & 3.9 & 4.9 \\
L & 1.0 & 1.0 & 1.0
\end{tabular}

a) Estimated preference parameters $\left(\hat{\lambda_{l k}}\right.$ 's $)$

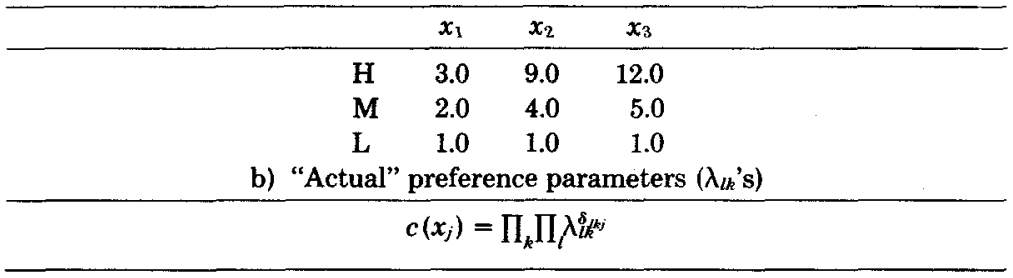

predictive capability relative to the ordinal theory normally used in conjoint analysis.

The empirical problem is to design a mix of telecommunications technology for use in a small research community. Scientific research requires close effective communication among scientists, but in many government laboratories cooperating scientists and managers find themselves in buildings two to three miles apart. Furthermore, laboratories, such as Los Alamos Scientific Laboratory (LASL) in New Mexico, do much of their work for federal agencies, and there is a strong need for effective communication with managers and policy makers in Washington, D.C. Currently, the most common means of communication at LASL is telephone (39\% in LASL) and personal visit (58\% in LASL), with only a small percentage (3\% in LASL) of the interactions using other means. The National Science Foundation would like to enhance communication among the scientists, managers, and policy makers with an improved system which is more effective than telephone for technical communication, yet more efficient than personal visits in terms of cost, time, and 
energy. Among the options being considered are closed-circuit television, telecopiers (facsimile transfer devices or teletypewriters), and narrowband televideo systems (an attachment to the telephone which transmits still pictures over voice-grade telephone lines). Since the laboratories have limited budgets, each laboratory would like to implement the communications system that would be most cost-effective. To do this the laboratories need to know how scientists and managers would react to the various systems.

\section{Study Design}

To address this problem, we used the normative methodology described in Hauser and Urban [17] to identify the relevant dimensions that describe communications options and to identify the relative importances of these dimensions. These dimensions form the basis for the CSPC questions used in the estimation of preference functions.

First, consumer focus groups were run and analyzed to produce an indication of the choice process, consumer semantics, and a set of 25 attribute scales to characterize consumer reactions to communication technology. Based on the focus groups and on previous research in the area of communications, a mail-back questionnaire was designed and implemented in which consumers rated telephone, personal visit, and the three new communications options (one-page concept statements) on the 25 attribute scales. Factor analysis of the response revealed two perceptual dimensions labeled "ease of use" and "effectiveness." Ease of use correlates with the ability to find the right person, save time, eliminate paperwork, and get a quick response as well as saving hassle, planning, time, and cost; effectiveness correlates with the ability to exchange scientific and technical information, persuade people, convey all forms of information, control the impression you want to make, monitor people, operations, and equipment, yield a high level of human interaction, solve problems, express feelings, and enhance idea development.

Scientific and managerial communication is complex, and it is probable that the communications needs would vary by individual depending upon his or her requirements. Thus, to accurately analyze preferences for communications options, we need to stratify by use scenario (purpose, distance between communicators, relation of communicators, etc.) and estimate preference functions within each category. CSPC questions were used to measure these preference functions.

\section{Consumer Measurement}

Based on the results of the mail questionnaire, a preference assessment questionnaire was designed to measure the CSPC data needed for the preference functions. This questionnaire was then implemented via an 
interactive computer interviewing system (Shugan and Hauser [47]) to scientists and managers at LASL and practicing managers enrolled in Northwestern University's Manager's Program (evening work toward a master's in management). The questionnaire contained six sections: (1) warm-up questions; (2) questions to establish a scenario for usage; (3) consumer rating of effectiveness and ease of use for telephone, personal visit, and for the concept statements; (4) the CSPC questions; (5) preference ranking and usage intent for the existing products and the concepts; and (6) various personal and demographic questions and comments.

Section 3 of the questionnaire was included to acquaint consumers with the measurement scales for effectiveness and ease of use and to provide us with their perceptions of each product or concept. Section 5

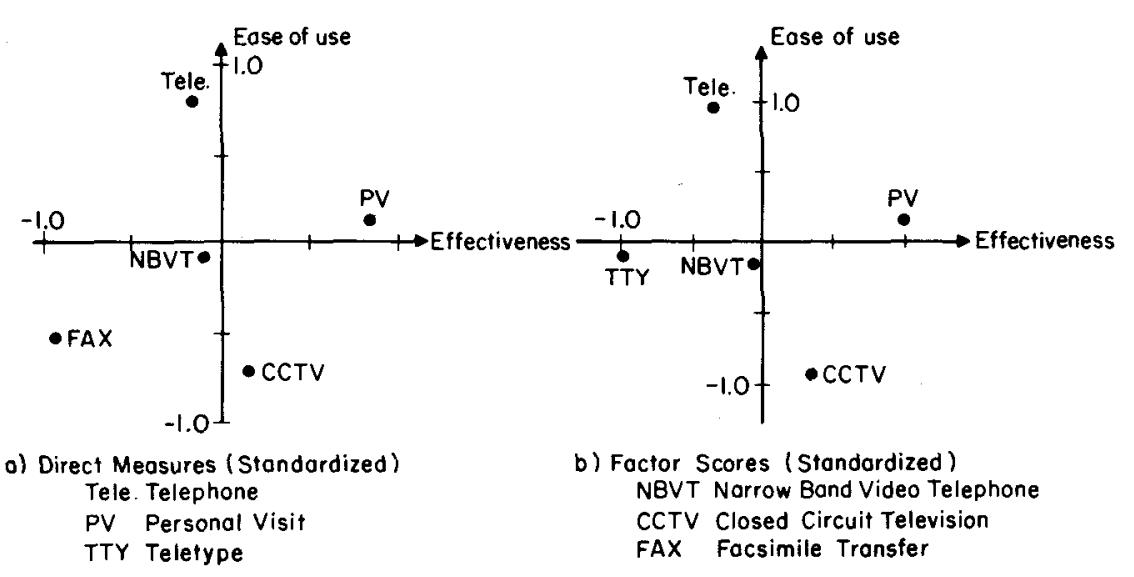

Figure 2. Perceptual maps of communications options.

provided preference measures for the actual products and concepts. These measures were used to validate predictions made by each theory based on CSPC questions.

The complete questionnaire contained 96 questions, including 16 CSPC questions, and took about 15-30 minutes to complete. The administration cost, including on-line hookup, was about $\$ 1.00$ per respondent on a CDC6400 ( $\$ 510$ per cpu hour). The comparative results reported below are based on the exploratory phase of the study where the directions of the CSPC question were left ambiguous. The sample was 41 practicing managers.

\section{Results}

Figure 2 gives the perceptual maps positioning of the five stimuli based on factor scores (mail survey) and the direct measures (preference sur- 
vey). The close agreement of the relative stimuli position in the two maps supports the direct measures of effectiveness and ease of use as sufficient for the preference analysis. (Note the stimuli "teletype terminals," used in the mail questionnaire, was revised to "facsimile transfer devices" in the preference questionnaire.)

Since we did not know either $\sigma$ or $\rho$, we need the $F$-tests in the exploratory analyses with the 0.05 -level cutoff of $K_{0.05}(0)=4.1$ (the $Q^{Y}$ and $Q^{R}$ statistics each have three and eight degrees of freedom). When neither ratio or interval theories were rejected, consumers were assigned to the theory with the largest $Q$.

The ordinal test identified $83 \%$ of the consumers as using at least ordinal theory at the 0.05 level (one of fewer transitivity violations) and $98 \%$ at the 0.15 level (two or fewer transitivity violations). The $F$-tests identified $44 \%$ of the consumers as using interval theory and $17 \%$ as using

TABLE IV

Percent of First Preferences Correctly Predicted

\begin{tabular}{lccc}
\hline \multirow{2}{*}{$\begin{array}{l}\text { Indicated } \\
\text { Theory }\end{array}$} & \multicolumn{3}{c}{ Estimation Procedure } \\
\cline { 2 - 4 } & Ordinal & Interval & Ratio \\
\hline Stochastic (7) & 0.6 & 0.7 & 0.7 \\
Ordinal (9) & 0.6 & 0.7 & 0.8 \\
Interval (18) & 0.5 & 0.7 & 0.7 \\
Ration (7) & 0.7 & 0.8 & 0.9 \\
\hline Total (41) & 0.57 & 0.72 & 0.74 \\
\hline
\end{tabular}

${ }^{a}$ Numbers in parentheses are sample sizes.

ratio theory at the 0.05 level $(61 \%$ of the consumers satisfied at least one intensity theory). No one was interval who was not at least ordinal.

To provide an alternative test for these identifications, we used each of the estimation procedures on each consumer and used the results to predict preference for the actual products (telephone, personal visit, NBV'T, CCTV, and FAX). This test is a predictive test because the models are estimated on CSPC questions relative to product attributes but used to predict preference for products not involved in the estimation. Based on the preceding results, we would (1) expect intensity theories to predict best overall, (2) expect the intensity theories to predict better on consumers identified as using an intensity theory, and (3) we would expect the ratio (interval) estimations to predict best for consumers identified as ratio (interval). Table IV reports the results of these tests. We have rounded to one decimal except for totals because of the extremely small sample sizes.

In general, Table IV supports these hypotheses. Intensity-based estimations were found superior to ordinal-based estimations. The estimations predict best for consumers identified as following ratio theory. Some 
anomalies do appear with interval theory, but one must be cautious when examining specific small sample entries which are not statistically different. It is interesting to note that the percentage of correct predictions for interval theory improve when we relax the confidence level on the $F$-test.

Table IV also suggests a potential robustness of intensity measures. Interval and ratio estimations exhibit similar overall predictive ability, and both intensity estimations outperform ordinal estimations, even if the intensity theories are rejected. There are small sample hypotheses which should be subjected to further testing.

To better understand Table IV, we analyzed individual predictions. Detailed analysis uncovered a fascinating finding. The improved prediction came from the intensity theories' ability to discriminate preferences between products which ordinal theory predicted as being equal in preferences. (E.g., the ratio theory might predict telephone as first

TABLE V

Comparison of Predicted Market Shares of Preference ${ }^{a}$

\begin{tabular}{|c|c|c|c|c|c|c|}
\hline \multirow{2}{*}{$\begin{array}{l}\text { Estimation } \\
\text { Procedure }\end{array}$} & \multicolumn{5}{|c|}{ Predicted Market Share (\%) } & \multirow{2}{*}{$\begin{array}{l}\text { Mean } \\
\text { absolute } \\
\text { error }\end{array}$} \\
\hline & Telephone & $\begin{array}{l}\text { Personal } \\
\text { visit }\end{array}$ & NBVT & CCTV & FAX & \\
\hline Ordinal & 28.0 & 38.2 & 13.8 & 13.0 & 8.1 & 6.90 \\
\hline Interval & 32.5 & 39.0 & 13.0 & 10.5 & 4.9 & 4.54 \\
\hline Ratio & 32.5 & 39.0 & 14.2 & 9.3 & 4.9 & 4.54 \\
\hline (Actual) & (36.6) & $(46.3)$ & (9.8) & (4.9) & $(2.4)$ & \\
\hline
\end{tabular}

${ }^{a}$ NBVT, narrow-band video telephone; CCTV, closed circuit television; FAX, facsimile transfer device.

preference, while ordinal theory would predict that telephone is tied with NBVT for first preference.) We have found by discussion with other researchers that others have also observed this limitation when estimating ordinal preference functions. Thus ordinal analysis is a "correct" representation of consumer preference, but the intensity theories produce preference functions which allow discrimination among products at the individual level by extracting additional information from individual responses.

Table V compares the techniques on their ability to correctly predict market preference shares. Again the intensity estimations provide improved predictions over the ordinal estimations. The overprediction of preference for the concepts over actual products results from consumers tending to favor an existing alternative over a new concept when predicted preference indicated indifference. Future research will expand the theories to include this "preference inertia" effect (Neslin [39]). Empirically, when the models are applied to existing alternatives only, the mean absolute error is reduced to $1.4 \%$ for both intensity theories. Furthermore, the relative comparisons remain the same. 
Predictive accuracy is important for the evaluation of alternative products; but to improve the design of new products, managers need diagnostic information to help them understand consumer preferences and thus design improved products (Little [31], Hauser and Urban [17]). Although preference functions are estimated at the individual level, it is useful to present summary statistics (mean, variance, median, interquartile range) to represent the target population. Figure 3 is a graph of the average preference functions. (The "utility" of effectiveness is scaled 0 to 1 for consistent comparison.) Note that although the average individual functions (ratio, interval, ordinal) are relatively close, the ordinal theory overestimates the importance of ease of use relative to intensity theories. The interpretations are quite intuitive with decreasing returns on effectiveness and a slight threshold on ease of use. These results are consistent
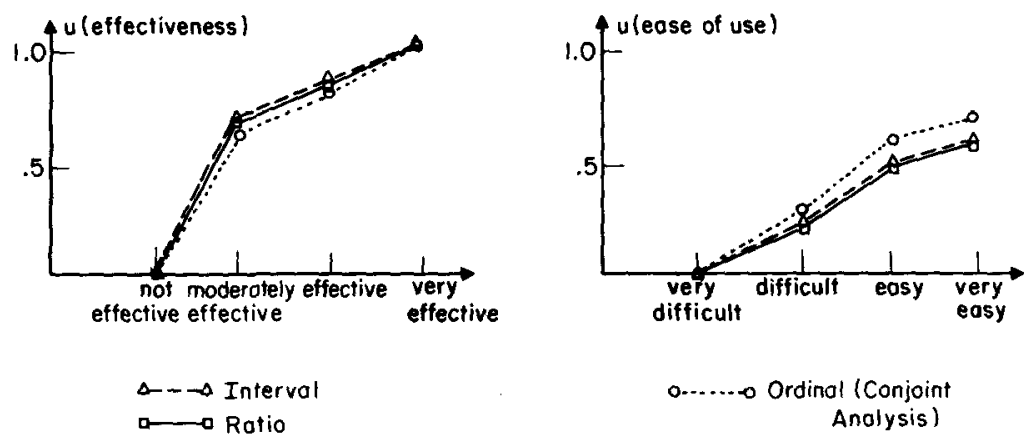

Figure 3. Comparison of preference functions.

with two preceding conclusions. First, as expected, ordinal theory provides "correct" representations of consumer preference and, second, the improvements achieved by intensity of preference theories result from improved discrimination among individual preference functions.

Thus the empirical evidence supports the theoretical development. Furthermore, this case illustrates how the theories can be used in an actual marketing research environment to provide managerially useful diagnostics and predictive capabilities. Even with the CSPC question left unspecified, predictions and diagnostics improved over the ordinal theory and estimation used in standard conjoint analysis or utility theory. We expect stronger evidence with more directed questions.

\section{SUMMARY AND FUTURE DIRECTIONS}

Consumer preference functions are important for many marketing research applications. The use of intensity measures such as CSPC or GPC has the potential to improve the accuracy and/or decrease the measurement burden of preference measurement. This paper provided a 
general structure, identification tests, and estimation procedures for preference functions based on intensity measures. Furthermore, the empirical implementation demonstrates how the theoretical development translates into a practical technique with potentially many managerial advantages.

This paper addresses many issues of intensity measurement, but many issues remain. Within the structure, further theoretical and practical development is necessary for the hybrid model and stochastic Theory I. The statistical models should be further investigated (e.g. does Theorem 2 ever support interval theory if the data is merely ordinal). Empirical work is needed to provide further guidelines for the magnitude of the error variance. Methods for estimating more complex utility functions

TABLE A1-I

\begin{tabular}{|c|c|c|c|c|}
\hline \multirow{2}{*}{$E_{0}$} & \multicolumn{2}{|c|}{$p=(14 / 27)$} & \multicolumn{2}{|c|}{$p=(1 / 24)$} \\
\hline & Theorem 7 & Simulation & Theorem 7 & Simulation \\
\hline 0 & 0.003 & 0.005 & 0.100 & 0.101 \\
\hline 1 & 0.028 & 0.040 & 0.363 & 0.348 \\
\hline 2 & 0.122 & 0.141 & 0.678 & 0.653 \\
\hline 3 & 0.324 & 0.336 & 0.886 & 0.874 \\
\hline 4 & 0.595 & 0.595 & 0.973 & 0.971 \\
\hline 5 & 0.829 & 0.825 & 0.996 & 0.997 \\
\hline 6 & 0.955 & 0.953 & 1.000 & 1.000 \\
\hline 7 & 0.994 & 0.994 & 1.000 & 1.000 \\
\hline 8 & 1.000 & 1.000 & 1.000 & 1.000 \\
\hline
\end{tabular}

must be found. The robustness hypothesis requires empirical testing and perhaps a theoretical explanation. The robustness of the tests to the normality assumption should be shown. Preference inertia can be added to the theories and models. In conclusion, intensity measurement was motivated by our expectation that intensity questions give more information per question. 'This hypothesis was supported. But intensity questions may be more difficult for the consumer to answer than ordinal questions. It remains an empirical question whether, in a given time period, more information can be gathered with intensity or with ordinal questions.

\section{APPENDIX 1: SIMULATIONS FOR ORDINAL THEORY TEST}

In Theorem 7 we derived a Bernoulli test for independent ordinal transitivity tests and conjectured that it was a good approximation for interdependent tests (Table A1-I). To support this conjecture, we randomly generated data for the experimental design in Table II and com- 
pared the observed violations to those predicted by Theorem 7 . The simulation sample size was 70,000 consumers for each comparison generated by an HP3000 random number generator.

\section{APPENDIX 2: TEST FOR STOCHASTIC THEORY I}

The assumptions for stochastic theory implies if $p=a_{i j} / S$ where $a_{i j}+$ $a_{j i}=S$ then $p_{j}=p_{j+1}$ for all positive integers $s$. i.e.

This implication indicates $a_{i j}, a_{j k}$ and $a_{i k}$ are distributed binomially,

$$
B\left(a_{i j} ; \theta\right)=\left(\begin{array}{c}
S \\
a_{i j}
\end{array}\right) \theta^{a_{i j}}(1-\theta)^{a_{j i}}
$$

where $0 \leq \theta \leq 1$. Let $p, q$, and $r$ be the probabilities that generated $a_{i j}$, $a_{j k}$, and $a_{i k}$, then Axiom $3,(p /(1-p))(q /(1-q))(r /(1-r))=1$, hence,

$$
r=[p q /(1-p-q+2 p q)] \text {. }
$$

The likelihood ratio $\lambda$ is given by

$$
\lambda=\frac{p^{a_{i j}}(1-p)^{a_{j i}} q^{a_{i j}}(1-q)^{a_{j i}} r^{a_{i k}}(1-r)^{a_{k i}}}{p^{a_{i j}}(1-p)^{a_{j i}} q^{a_{i j}}(1-q)^{a_{i j}} R^{a_{i k}}(1-R)^{a_{k i}}}=\left[\frac{r(1-R)}{R(1-r)}\right]^{a_{i k}}\left(\frac{1-r}{1-R}\right)^{S}
$$

where $R$ is independent of $p$ and $q, r$ is given by (A.1), hence, $a_{i k}$ is our test statistic.

Then, for any given $R$, the Neyman-Pearson lemma (Wilks [56]) states the most powerful test of size $\alpha$ would be to reject stochastic theory if and only if $\lambda<k$, where $\alpha=\sum_{n=k}^{S}\left(\begin{array}{l}S \\ n\end{array}\right) r^{a_{i j}}(1-r)^{a_{i j}}$. However, to use Axiom 3, it is necessary to estimate $p$ and $q$. The maximum likelihood estimators for $p$ and $q$ are found by interatively solving (perhaps by a Fibonacci search [35]), the following single parameter equation:

$$
\left(a_{j k}-q\right) p^{2}+\left(q^{2}-a_{j k}\right) p+a_{i j} q(1-q)=0
$$

where

$$
\begin{aligned}
q & =(-A+p \sqrt{B}) / C \\
A & =\left(1+2 a_{i k}\right) p^{2}-\left(3 a_{i j}-5 a_{i k}\right) p+\left(2 a_{i j}+2 a_{i k}\right) \\
B & =\left[\left(1+2 a_{i k}\right) p+\left(a_{i j}-a_{i k}\right)\right]^{2}-4\left(a_{i j}+a_{i k}\right) \\
C & =\left(-4-8 a_{i k}\right) p^{2}+\left(4 a_{i j}+8 a_{i k}\right) p+\left(-2 a_{i j}-2 a_{i k}\right) .
\end{aligned}
$$

When employing this method of estimation, our experience has identified problems involving global optimality and boundary solutions. One must ensure against convergence to local optimal and infeasible values for $p(p>1$ or $p<0)$.

\section{APPENDIX 3: ILLUSTRATIVE EXAMPLE}

This example illustrates how the statistical tests and estimations developed in the paper are applied in practice. We also illustrate how the Hauser-Urban [17] methodology was employed. 
We arbitrarily (randomly) select the very first respondent in our sample and demonstrate, in detail, the calculations for this respondent.

As noted earlier, focus groups identified different product attributes as important in decision-making. Table A3-I illustrates how these attributes were cataloged and typical focus group quotes referring to these attributes.

The results of the perceptual questioning were factor analyzed. As we can see from the factor loadings in Table A3-III, there are two perceptual dimensions, "efficacy" and "ease-of-use." We, therefore, ask respondents to allocate 100 chips between hypothetical products specified by these two dimensions. We used an interactive query system [47] for this purpose. Chips allocations for the first respondent for the $16 \mathrm{CSPC}$ questions were:

$(60,40),(10,90),(60,40),(10,90),(90,10),(40,60)$, $(20,80),(1,99),(70,10),(10,90),(99,1),(70,30)$, $(70,30),(80,20),(40,60),(30,70)$.

For example, in the first question the respondent allocated 60 chips to the first product and 40 chips to the second product (see Table A3-IV). Although Respondent 1 tended to round off the chip allocations, this did not happen for all respondents.

Our experimental design matrix identifying transitivity tests for these 16 paired comparison questions had 8 embeded transitivity tests. That matrix follows:

$$
M=\left[\begin{array}{rrrrrrrrrrrrrrrr}
1 & 0 & 1 & 0 & 0 & 1 & 0 & 0 & 0 & 0 & 0 & 0 & 0 & 0 & 0 & 0 \\
1 & 0 & 0 & -1 & -1 & 0 & 0 & 0 & 0 & 0 & 0 & 0 & 0 & 0 & 0 & 0 \\
0 & 1 & -1 & -1 & 0 & 0 & 0 & 0 & 0 & 0 & 0 & 0 & 0 & 0 & 0 & 0 \\
0 & 0 & 0 & 0 & 0 & 0 & 1 & 1 & 1 & 0 & 0 & 0 & 0 & 0 & 0 & 0 \\
0 & 0 & 0 & 0 & 0 & 0 & 0 & 0 & 0 & 0 & 0 & 1 & 1 & -1 & 0 & 0 \\
0 & 0 & 0 & 0 & 0 & 0 & 1 & 0 & 0 & 1 & 1 & 0 & 0 & 0 & 0 & 0 \\
0 & 0 & 0 & 0 & 0 & 0 & 1 & 0 & 0 & 0 & 0 & 0 & 0 & 0 & 1 & -1 \\
0 & 0 & 0 & 0 & 0 & 0 & 0 & 1 & 0 & -1 & 0 & 0 & 0 & -1 & 0 & 0
\end{array}\right]
$$

We now generate a matrix $W$ such that (1) $M W^{\prime}=0,(2) W$ has exactly 3 nonzero elements per row each being \pm 1 and (3) $W$ is of maximum rank. The $W$ matrix was found by exhaustive enumeration. $W$ was unique and is given as follows:

$$
W=\left[\begin{array}{rrrrrrrrrrrrrrrr}
0 & -1 & 0 & -1 & 1 & 0 & 0 & 0 & 0 & 0 & 0 & 0 & 0 & 0 & 0 & 0 \\
1 & 0 & 0 & 0 & 1 & -1 & 0 & 0 & 0 & 0 & 0 & 0 & 0 & 0 & 0 & 0 \\
1 & 0 & -1 & 1 & 0 & 0 & 0 & 0 & 0 & 0 & 0 & 0 & 0 & 0 & 0 & 0
\end{array}\right]
$$

We did not know the measurement error variance and therefore employed the $F$-tests. For interval theory, the data vector of differences, $d^{I}$ 
TABLE A3-I

FUndamental AtTributes ${ }^{a}$

\begin{tabular}{|c|c|c|c|c|c|}
\hline $\begin{array}{l}\text { Generic } \\
\text { Name }\end{array}$ & Description & $\begin{array}{l}\text { Engineering } \\
\text { Variable }\end{array}$ & $\begin{array}{l}\text { Marketing } \\
\text { Concept }\end{array}$ & Quote & $\begin{array}{c}\text { Agree- } \\
\text { Disagree } \\
\text { Statement }\end{array}$ \\
\hline $\begin{array}{l}\text { Group } \\
\text { interaction }\end{array}$ & $\begin{array}{l}\text { Teamwork, several } \\
\text { speakers, } \\
\text { multiperson } \\
\text { interaction, } \\
\text { collaboration }\end{array}$ & Symbiotic & Teamwork, synergic & $\begin{array}{l}\text { "Group work," "team } \\
\text { effort," "teamwork" }\end{array}$ & $\begin{array}{l}\text { CCTV would be good } \\
\text { for group discusson }\end{array}$ \\
\hline Speed & $\begin{array}{l}\text { Expedient, quick, } \\
\text { information per } \\
\text { minute, rapid } \\
\text { transmission, waiting } \\
\text { time, prompt }\end{array}$ & $\begin{array}{l}\text { Real time, frame } \\
\text { rate, delay } \\
\text { time }\end{array}$ & $\begin{array}{l}\text { Time spent, takes } \\
\text { forever }\end{array}$ & $\begin{array}{l}\text { "Crisis calls," "frame } \\
\text { rate" }\end{array}$ & $\begin{array}{l}\text { CCTV would save me } \\
\text { time }\end{array}$ \\
\hline Security & $\begin{array}{l}\text { Confidentiality, } \\
\text { classified }\end{array}$ & Security level & & $\begin{array}{l}\text { "Need approval," } \\
\text { "security clearance" }\end{array}$ & $\begin{array}{l}\text { Security would be no } \\
\text { problem with CCTV }\end{array}$ \\
\hline $\begin{array}{l}\text { Preparation } \\
\text { time }\end{array}$ & $\begin{array}{l}\text { Ready, groundwork, } \\
\text { convenient to use, } \\
\text { commitment, } \\
\text { foundation, planning }\end{array}$ & Setup cost & $\begin{array}{l}\text { Preparation, } \\
\text { preliminaries }\end{array}$ & $\begin{array}{l}\text { "Preparation } \\
\text { involved," "easy to } \\
\text { use" }\end{array}$ & $\begin{array}{l}\text { I would need to plan } \\
\text { far in advance to use } \\
\text { CCTV }\end{array}$ \\
\hline
\end{tabular}

${ }^{a}$ Consumer perceptions on these attributes were now measured with 25 agree-disagree statements. Three such statements are illustrated in Table A3-II. CCTV, closed circuit television. 
TABLE A3-II Sample AGReE-Disagree Questions

\begin{tabular}{|c|c|c|c|c|c|}
\hline \multicolumn{6}{|c|}{$\begin{array}{l}\text { My Rating of Narrow-band Video Telephone (NBVT) } \\
\text { (for the Interaction I Described Earlier) }\end{array}$} \\
\hline & $\begin{array}{l}\text { Strongly } \\
\text { disagree }\end{array}$ & Disagree & $\begin{array}{l}\text { Neither } \\
\text { agree } \\
\text { nor } \\
\text { disagree }\end{array}$ & Agree & $\begin{array}{l}\text { Strongly } \\
\text { agree }\end{array}$ \\
\hline $\begin{array}{l}\text { 1. By NBVT I could not effec- } \\
\text { tively exchange scientific and } \\
\text { technical information. }\end{array}$ & [ ] & [ ] & {$\left[\begin{array}{ll}1 \\
\text { ] }\end{array}\right.$} & {$[1$} & [ ] \\
\hline $\begin{array}{l}\text { 2. I could always find and reach } \\
\text { the person I want by NBVT. }\end{array}$ & [ T & {$[\quad]$} & [ ] & {$[1$} & {[]} \\
\hline 3. NBVT would save me time. & [ ] & [ ] & 「 1 & {$[1]$} & [ ] \\
\hline
\end{tabular}

was computed from the preceding chip allocations. That vector follows: $d^{I}$

$$
=\left[\begin{array}{llllllllllllllll}
20 & -80 & 20 & -80 & 80 & -20 & -60 & -98 & 40 & -80 & 98 & 40 & 40 & 60 & -20 & -40
\end{array}\right]^{\prime} \text {. }
$$

We compute the vectors $\nu$ and $\eta$. The vector $\nu$ represents the vector of transitivity tests as determined by the experimental design. The vector

TABILE A3-III

Factor Loadings on Fundamental Attributes

\begin{tabular}{lcc}
\hline & Efficacy & Ease of Use \\
\hline 1. Effective information exchange & 0.77 & 0.17 \\
2. Find right person & 0.25 & 0.43 \\
3. Save time & 0.17 & 0.48 \\
4. Not need visual & 0.39 & 0.16 \\
5. Get trapped & 0.33 & 0.21 \\
6. Eliminate paperwork & 0.31 & 0.43 \\
7. Persuade & 0.69 & 0.20 \\
8. Focus on issues & 0.04 & 0.08 \\
9. All forms information & 0.65 & 0.18 \\
10. Real hassle & 0.11 & 0.83 \\
11. Control impression & 0.56 & 0.07 \\
12. Security & 0.18 & 0.11 \\
13. Plan in advance & 0.23 & 0.44 \\
14. Eliminate red tape & 0.00 & 0.21 \\
15. Monitor people, operations, experiments & 0.65 & 0.15 \\
16. Interaction & 0.78 & 0.05 \\
17. Solve problems & 0.55 & 0.27 \\
18. Express feelings & 0.66 & 0.17 \\
19. Misinterpret & 0.49 & 0.00 \\
20. Group discussion & 0.75 & 0.05 \\
21. Inexpensive & 0.27 & 0.52 \\
22. Quick response & 0.07 & 0.71 \\
23. Enhance idea development & 0.77 & 0.09 \\
24. Commitment & 0.44 & 0.32 \\
25. Maintain contact & 0.50 & 0.52 \\
\hline
\end{tabular}


TABLE A3-IV

First Constant Sum Paired Comparisons (CSPC) Question

\begin{tabular}{ll}
\hline \multicolumn{1}{c}{ Product A } & \multicolumn{1}{c}{ Product B } \\
\hline Effective & Moderately effective \\
Difficult to use & Easy to use \\
$? 60,40$ & \\
\hline
\end{tabular}

$\eta$ represents independent triplets or randomly selected transitivity tests. These vectors follow:

$$
\begin{aligned}
\nu & =M d^{I}=\left[\begin{array}{llllllll}
20 & 20 & -20 & -118 & 20 & -42 & -40 & -78
\end{array}\right]^{\prime} \\
\eta & =W d^{I}=\left[\begin{array}{llll}
240 & 120 & -80
\end{array}\right]^{\prime}
\end{aligned}
$$

For interval theory the adjusted sum of squared error, $\mathscr{E}^{I}$, is given as follows:

$$
\begin{aligned}
& =\nu^{\mathscr{E}}\left(M M^{\prime}\right)^{-1} \nu / s^{2} \\
& =\left(\frac{1}{s^{2}}\right)\left[\begin{array}{c}
20 \\
20 \\
-20 \\
-118 \\
20 \\
-42 \\
-40 \\
-78
\end{array}\right]\left[\begin{array}{rrrrrrrr}
.50 & -.25 & .25 & .00 & .00 & .00 & .00 & .00 \\
-25 & .50 & -.25 & .00 & .00 & .00 & .00 & .00 \\
.25 & -.25 & .50 & .00 & .00 & .00 & .00 & .00 \\
.00 & .00 & .00 & .55 & .10 & -.25 & -.10 & -.30 \\
.00 & .00 & .00 & .10 & .40 & -.10 & .00 & -.20 \\
.00 & .00 & .00 & -.25 & -.10 & .55 & -.10 & .30 \\
.00 & .00 & .00 & -.10 & .00 & -.10 & .40 & -.00 \\
.00 & .00 & .00 & -.30 & -.20 & .30 & -.00 & .60
\end{array}\right]\left[\begin{array}{c}
20 \\
20 \\
-20 \\
-118 \\
20 \\
-42 \\
-40 \\
-78
\end{array}\right] \\
& =6484 / s^{2} .
\end{aligned}
$$

If we knew the measurement variance, $s^{2}$, we could test to see if $6484 / s^{2}$ was significant. However, we do not. Therefore, we compute the noncentral $\chi^{2}$ variate $\eta^{\prime}\left(W W^{\prime}\right)^{-1} \eta / s^{2}$.

We find,

$$
\begin{aligned}
\left(1 / s^{2}\right) \eta^{\prime}\left(W W^{\prime}\right)^{-1} \eta & =\left[\begin{array}{c}
240 \\
120 \\
-80
\end{array}\right]\left[\begin{array}{ccc}
.5 & -.25 & .25 \\
-.25 & .5 & -.25 \\
.25 & -.25 & .5
\end{array}\right]\left[\begin{array}{c}
240 \\
120 \\
-80
\end{array}\right] / s^{2} \\
& =20,000 / s^{2} .
\end{aligned}
$$

We now employ Theorem 2 and find

$$
Q^{I}=\left(s^{2} \eta^{\prime}\left(W W^{\prime}\right)^{-1} \eta / 3\right) /\left(s^{2} \nu^{\prime}\left(M M^{\prime}\right)^{-1} \nu / 8\right)=(8 / 3)(20000 / 6484)=8.22 \text {. }
$$

Using Theorem 2 , we employ a central $F$-test. The cutoff values for the $F$-distribution are 2.92 for $\alpha=0.1$ and 4.07 for $\alpha=0.05$. Hence, $Q^{I}$ is 
significant for both $\alpha=0.1$ and $\alpha=0.05$. Interval theory is therefore supported.

For ratio theory, $d^{R}$ represents the vector of log-ratios and is given as follows (values are rounded to nearest integer for display purposes):

$$
d^{R}=\left[\begin{array}{llllllllllllllll}
0 & -2 & 0 & -2 & 2 & -0 & -1 & -5 & 1 & -2 & 5 & 1 & 1 & 1 & -0 & -1
\end{array}\right]^{\prime} .
$$

We continue by computing the value of $Q^{R}$ as expressed by Theorem 5 .

$$
\begin{aligned}
& v=\left[\begin{array}{llllllll}
.405 & .405 & -.405 & -5.134 & .308 & 1.012 & -.944 & -3.784
\end{array}\right]^{\prime} \\
& \nu^{\prime}\left(M M^{\prime}\right)^{-1} \nu \\
& =\left[\begin{array}{r}
.405 \\
.405 \\
-.405 \\
-5.13 \\
.308 \\
1.01 \\
-.944 \\
-3.78
\end{array}\right]\left[\begin{array}{rrrrrrrr}
.50 & -.25 & .25 & .00 & .00 & .00 & .00 & .00 \\
-.25 & .50 & -.25 & .00 & .00 & .00 & .00 & .00 \\
.25 & -.25 & .50 & .00 & .00 & .00 & .00 & .00 \\
.00 & .00 & .00 & .55 & .10 & -.25 & -.10 & -.30 \\
.00 & .00 & .00 & .10 & .40 & -.10 & .00 & -.20 \\
.00 & .00 & .00 & -.25 & -.10 & .55 & -.10 & .30 \\
.00 & .00 & .00 & -.10 & .00 & -.10 & .40 & -.00 \\
.00 & .00 & .00 & -.30 & -.20 & .30 & -.00 & .60
\end{array}\right]\left[\begin{array}{r}
.405 \\
.405 \\
-.405 \\
-5.13 \\
.308 \\
1.01 \\
-.944 \\
-3.78
\end{array}\right] \\
& =12.16 \\
& \eta^{\prime}\left(M M^{\prime}\right) \eta^{-1}=\left[\begin{array}{r}
6.591 \\
3.008 \\
-2.197
\end{array}\right]\left[\begin{array}{ccc}
.5 & -.25 & .25 \\
-.25 & .5 & -.25 \\
.25 & -.25 & .5
\end{array}\right]\left[\begin{array}{r}
6.591 \\
3.008 \\
-2.197
\end{array}\right] \\
& =14.81 \\
& Q^{R}=(8 / 3)(14.81 / 12.16) \\
& =3.25 \text {. }
\end{aligned}
$$

Noting the same cutoff values of 2.92 and 4.07, we see $Q^{R}$ is significant at the 0.1 level but not the 0.05 level. Therefore, ratio theory is only supported at the 0.1 level.

Finally, for ordinal theory we find $E^{0}=0$. Hence, ordinal theory is supported at any $\alpha$ level. If, however, $E^{0}$ was greater than 0 , then Appendix 1 would provide cutoff values for judging significance. The results for this respondent (Table A3-V) can be compared to the results

TABLE A3-V

First Person Example Results

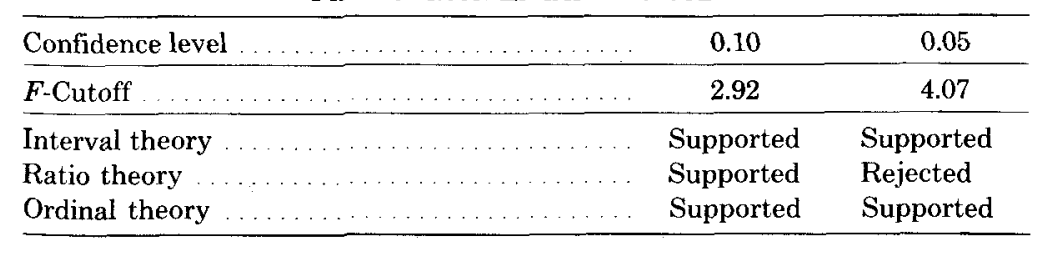


TABLE A3-VI

Total SAMPLE

\begin{tabular}{|c|c|c|}
\hline Confidence level & 0.10 & 0.05 \\
\hline Interval supported & $63.4 \%$ & 58.5 \\
\hline Ratio supported & $53.7 \%$ & 48.8 \\
\hline Interval or ratio & $63,4 \%$ & 61.0 \\
\hline Ordinal supported & $98.0 \%^{a}$ & 83.0 \\
\hline
\end{tabular}

${ }^{a}$ Due to integer requirements the ordinal theory corresponds to the 0.15 level.

in Table A3-VI which represent total sample statistics. Note that the ratio test was able to reject ratio theory at the 0.05 level even though the respondent had no ordinal transitivity violations.

We now proceed to estimate this respondent's preference functions. For interval theory, we employ Equations 6 and 7. Using Theorem 3, we solve the following linear program.

$$
\begin{aligned}
& \text { minimize } \sum_{t=1}^{16} e_{t} \\
& \text { subject to } e_{t} \geq-E_{t} \text { for } t=1, \cdots, 16 \\
& e_{t} \geq E_{t} \quad \text { for } \quad t=1, \cdots, 16 \\
& \lambda_{11} \leq \lambda_{21} \leq \lambda_{31} \leq \lambda_{41} \text { and } \lambda_{12} \leq \lambda_{22} \leq \lambda_{32} \leq \lambda_{42}
\end{aligned}
$$

where for example,

$$
\begin{aligned}
& E_{1}=(60-40)-\left[\left(\lambda_{31}+\lambda_{22}\right)-\left(\lambda_{21}+\lambda_{32}\right)\right] \\
& E_{2}=(10-90)-\left[\left(\lambda_{11}+\lambda_{42}\right)-\left(\lambda_{41}+\lambda_{12}\right)\right], \text { etc. }
\end{aligned}
$$

and, $\lambda_{l 1}=$ the utility of the $l$ th level of effectiveness $(1=$ not effective, 2 $=$ moderately effective, $3=$ effective, $4=$ very effective) and $\lambda_{l 2}=$ the utility of the $l$ th level of ease-of-use $(1=$ very difficult, $2=$ difficult, $3=$ easy, $4=$ very easy).

The results of this linear program for the first respondent for ordinal, interval, and ratio theories are given in Table A3-VII.

TABLE A3-VII

RESPONDENT UTLITIES

\begin{tabular}{cccccccc} 
& \multicolumn{3}{c}{ Effectiveness } & & \multicolumn{3}{c}{ Ease-of-Use } \\
\cline { 2 - 4 } \cline { 6 - 7 } & Ordinal & Interval & Ratio $^{a}$ & & Ordinal & Interval & Ratio $^{\alpha}$ \\
\hline 1 & 0.0 & 0 & 0.00 & 0.0 & 0 & 0.00 \\
2 & 1.5 & 90 & 2.63 & 1.0 & 30 & 1.24 \\
3 & 3.0 & 140 & 4.31 & 2.0 & 80 & 2.92 \\
4 & 3.0 & 150 & 5.14 & 2.5 & 90 & 3.35 \\
\hline
\end{tabular}

"Ratio utilities are expressed as logarithms. 
TABLE A3-VIII

Respondent Ratings

\begin{tabular}{lll}
\hline Product $^{a}$ & Effectiveness & \multicolumn{1}{c}{ Ease-of-Use } \\
\hline TEL & Moderate (2) & Easy (3) \\
PV & Effective (3) & Difficult (2) \\
NBVT & Effective (3) & Easy (3) \\
CCTV & Not very (1) & Very difficult (1) \\
FAX & Moderate (2) & Easy (3) \\
\hline
\end{tabular}

"TEL, telephone; PV, personal visit; NBVT, narrow-band video telephone; CCTV, closed circuit television; FAX facsimile transfer device.

${ }^{b}$ Numbers denote the attribute level.

TABLE A3-IX

RESPONDENT UTILITIES ${ }^{a}$

\begin{tabular}{lccccr}
\hline Theory & TEL & PV & NBVT & CCTV & FAX \\
\hline Ordinal & 3.5 & 4.0 & 5.0 & 0 & 3.5 \\
Interval & 170.0 & 170.0 & 220.0 & 0 & 170.0 \\
Ratio & 5.55 & 5.55 & 7.23 & 0 & 5.5 \\
\hline
\end{tabular}

${ }^{a}$ Abbreviations as in Table A3-VIII.

We next asked the respondent to rate telephone (TEL), personal visit (PV), narrow-band video telephone (NBVT), closed circuit television (CCTV), and facsimile transfer devices (FAX). Summary descriptions of the product concepts are given in Table A3-XI. The respondents were given one page verbal descriptions of these concepts. For example Table A3-XII gives the NBVT concept. The first respondent's ratings are given in Table A3-VIII.

We can now combine the ratings in Table A3-VIII with the utility values in Table A3-VII to derive composite consumer utility values for each product. The product utilities for the first respondent are shown in Table A3-IX.

The respondent was asked to rank the products in order of preference. These rankings are given in Table $\mathrm{A} 3-\mathrm{X}$ along with the rankings implied by Table A3-IX.

TABLE A3-X

Respondent's PREFERENCE RANking ${ }^{a}$

\begin{tabular}{llll}
\hline \multirow{2}{*}{ Actual } & \multicolumn{3}{c}{ Predicted } \\
\cline { 2 - 4 } & \multicolumn{1}{c}{ Ordinal } & \multicolumn{1}{c}{ Interval } & \multicolumn{1}{c}{ Ratio } \\
\hline 1. NBV'T & NBVT & NBVT & NBVT \\
2. TEL & PV & TEL-PV-FAX & TEL-PV-FAX \\
3. PV & FAX-TEL & CCTV & CCTV \\
4. FAX & CCTV & & \\
5. CCTV & & & \\
\hline
\end{tabular}

${ }^{a}$ Abbreviations as in Table A3-VIII. 
TABLE A3-XI

Features of Different Product Concepts

\begin{tabular}{|c|c|c|c|c|}
\hline Feature & $\begin{array}{l}\text { Narrow-band } \\
\text { Video } \\
\text { Telephone } \\
\text { (NBVT) }\end{array}$ & $\begin{array}{l}\text { Closed } \\
\text { Circuit } \\
\text { Television } \\
\text { (CCTV) }\end{array}$ & $\begin{array}{l}\text { Facsimile } \\
\text { Transfer } \\
\text { Device } \\
\text { (FAX) }\end{array}$ & $\begin{array}{c}\text { Teletype } \\
\text { Terminal } \\
\text { ('TTY) }\end{array}$ \\
\hline Video & Still pictures & Motion pictures & Hard copy & Typed page \\
\hline Availability & Your office & $\begin{array}{l}\text { Common area in } \\
\text { building }\end{array}$ & Your office & Your office \\
\hline \multicolumn{5}{|l|}{ Equipment for: } \\
\hline Reception & TV set & TV set & $\begin{array}{l}\text { Xerox-type } \\
\text { machine }\end{array}$ & Typewriter \\
\hline Transmission medium & $\begin{array}{l}1 \text { or } 2 \text { telephone } \\
\text { lines }\end{array}$ & Coaxial cable & Telephone line & Telephone line \\
\hline Picture resolution & Good & Fair to good & Excellent & Perfect \\
\hline Transmission time & $30 \mathrm{sec}$ & Immediate & $5 \min$ & 60 characters $/$ min \\
\hline Color & Optional & Yes & No & No \\
\hline Transmission cost & Telephone charge & $\$ 14 / \mathrm{min}$ & Telephone charge & Telephone charge \\
\hline Reservation & None & 4 days & None & None \\
\hline Usage limitation & None & 1 hour & None & None \\
\hline
\end{tabular}


TABLE A3-XII

EXample Concept Statement

\section{NARROW-BAND VIDEO TELEPHONE (NBVT)}

The narrow-band video telephone (NBVT) allows the user to transmit still pictures of himself, diagrams, drawings, written material, or equipment over ordinary telephone grade lines. Input is via a TV camera and display is on a standard TV set. The ability of NBVT to reproduce detail and shades of gray is similar to that of ordinary TV. In essence, NBVT is like transmitting viewgraphs, such as one might use with an overhead projector, over telephone lines. However, with NBVT the user can make pictures on the spot and edit these pictures before transmitting them.

No modification of the telephone is required. Units may be placed at any telephone location using either an acoustic coupler or a permanent jack. This makes NBVT portable, readily available, and allows individuals to use it in their own offices. Furthermore, because NBV'T transmits over ordinary telephone lines there are no additional costs associated with its use once the basic equipment has been purchased.

While NBVT can be used with only one telephone line, ordinarily an additional line is dedicated to voice transmission. Anything which can be viewed by a TV camera may be sent, but it takes 30 seconds to transfer a completely new picture. Once a picture is received, it is stored in memory and can be displayed indefinitely on a TV monitor. In addition, because the system is narrow-band, both audio and video transmission can be recorded on the stereo tracks of an ordinary audio cassette. With the cassette system it is possible to call and receive calls from an unattended terminal.

A variety of additional features are possible with NBVT. These are summarized below.

- Voice-grade crypto units can provide secure transmission when required

- Conferencing features of the audio telephone network

- Higher resolution and color

- Units are available which can display more than one stored picture simultaneously (on two monitors) or switch from one stored picture to another instantaneously.

You should assume that there is an NBVT unit in your office or laboratory and another $N B V T$ unit in the office of the person you wish to interact with. Assume that special units with the additional features listed above will be available on a reservation basis.

As we can see from Table A3-X, the intensity theories do slightly better than ordinal theory. Here they predict TEL, PV and FAX as equally preferred while ordinal theory incorrectly resolves the tie. By examining the results for other respondents, we find this case is not quite typical. Intensity theories do best. However, ordinal theory often predicts numerous ties while the intensity theories correctly resolve those ties. All three theories seem to have good predictive ability.

\section{ACKNOWLEDGMENTS}

Parts of the empirical study in Section 3 were funded by a grant from the National Science Foundation, DS176-09479, Stuart Meyer, principal investigator. We wish also to thank R. J. Taschek and T. Norris at Los Alamos Scientific Laboratory for their valuable assistance in the study. Paul Green, Dan Horsky, Subrata Sen, Al Silk, V. Srinivasan and Glen Urban provided important comments on earlier versions of this paper. 
We would also like to thank the referees who provided insightful guidance and provocative questions which led to major improvements in the theories, their use, and exposition.

\section{REFERENCES}

1. H. Abelman, "OPTISEP-A Package of Subroutines for Non-Linear Optimization," Vogel Computing Center, Northwestern University, Evanston, III., Document No. 322, July 1976.

2. J. Aitchison AND J. A. C. Brown, The Lognormal Distribution with Special Reference to Its Uses in Economics, Cambridge University Press, England, 1969.

3. F. BAss, "The Theory of Stochastic Preference and Brand Switching," $J$. Marketing Res. 11, 1-20 (1974).

4. J. Berkman, D. Brownstone, G. M. Duncan and D. McFadden, "Quail User's Manual," Urban Travel Demand Forecasting Project, University of California, Berkeley, Calif.; prepared for the National Science Foundation, revised January 1976.

5. S. R. CaRadus, Operator Theory of the Pseudo-Inverse, Queens University, Kingston, Ontario, Canada, 1974.

6. W. G. Cochran and G. M. Cox, Experimental Design, John Wiley \& Sons, New York, 1957.

7. C. Cohen, "SUMT Version 4: Sequential Unconstrained Minimization Techniques for Non-Linear Programming," Vogelback Computing Center, Northwestern University, Evanston, Ill., Document No. 200 (Rev. A), February 1975.

8. P. H. Farquhar, "A Survey of Multiattribute Utility Theory and Applications," Stud. Mgmt. Sci. 6, 59-89 (1977).

9. M. Friedman and L. J. Savage, "The Expected-Utility Hypothesis and the Measurability of Utility," J. Political Econ. 60, 463-474 (1952).

10. N. C. GIRI, Multivariate Statistical Inference, Academic Press, New York, 1977.

11. P. Green and M. Devita, "An Interaction Model of Consumer Utility," $J$. Consumer Res. 2, 146-153 (1975).

12. P. Green and V. Rao, Applied Multidimensional Scaling, Holt, Rinehart, \& Winston, New York, 1972.

13. P. E. Green and V. SRinivasan, "Conjoint Analysis in Consumer Behavior: Status and Outlook," J. Consumer Res. 5, 103-23 (1978).

14. P. E. Green And Y. Wind, Multiattribute Decisions in Marketing, Dryden Press, Hinsdale, Ill., 1973.

15. J. R. Hauser, "Testing the Accuracy, Usefulness, and Significance of Probabilistic Choice Models: An Information Theoretic Approach,"' Opns. Res. 26, 406-421 (1978).

16. J. R. Hauser, "Consumer Preference Axioms: Behavioral Postulates for Describing and Predicting Stochastic Choice," Mgmt. Sci. 24, 13 (1978).

17. J. R. Hauser and G. L. Urban, "A Normative Methodology for Modeling Consumer Response to Innovation," Opns. Res. 25, 574-619 (1977).

18. J. R. HAUSER AND G. L. URBAN, "Assessment of Attribute Importances and 
Consumer Utility Functions: von Neumann-Morgenstern Theory Applied to Consumer Behavior," J. Consumer Res. 5, 251-262 (1979).

19. I. N. Herstein ANd J. Milner, "An Axiomatic Approach to Measurable Utility," Econometrica 21, 291-297 (1953).

20. D. Horsky, S. SEn And S. Shugan, "Choice Models: A Review," Proceedings of the 1978 Educators' Conference, American Marketing Association, August 1978.

21. J. Huber and D. Sheluga, "The Analysis of Graded Paired Comparison in Marketing Research," Working Paper, Purdue University, Bloomington, Ind., May 1977.

22. N. E. JENSEN, "An Introduction to Bernoullian Utility Theory; I. Utility Functions," Swed. J. Econ. 69, 163-183 (1967).

23. R. M. Johnson, Multiple Discriminant Analysis Applications to Marketing Research, Market Facts, Inc., Chicago, January 1970.

24. R. M. Johnson, "Tradeoff Analysis of Consumer Values," J. Marketing Res. 11, 121-127 (1974).

25. R. M. Johnson, "Beyond Conjoint Measurement: A Method of Pairwise Trade-off Analysis," Proceedings of the Association of Consumer Research, Cincinnati, Ohio, October 1975.

26. R. L. KEeney, "Utility Functions for Multiattributed Consequences," $\mathbf{M g m t}$. Sci. 18, 276-287 (1972).

27. R. L. Keeney, "A Decision Analysis with Multiple Objectives: The Mexico City Airport," Bell J. Econ. Mgmt. Sci. 4, 101-117 (1973).

28. R. L. Keeney, "Multiplicative Utility Functions," Opns. Res. 22, 22-23 (1974).

29. R. L. KeEney AND H. RaIfFa, Decision Analysis with Multiple Conflicting Objectives, John Wiley \& Sons, New York, 1976.

30. D. H. Krantz, R. D. Luce, P. Suppes and A. Tversky, Foundations of Measurement, Academic Press, New York, 1971.

31. J. D. C. LitTLE, "Models and Managers: The Concept of a Decision Calculus," Mgmt. Sci. 16, 466-485 (1970).

32. R. D. LucE, Individual Choice Behavior, John Wiley \& Sons, New York, 1959.

33. R. D. LucE, "The Choice Axiom After Twenty Years," J. Math. Psychol. 15, 213-233 (1977).

34. R. D. Luce AND J. W. Tukey, "Simultaneous Conjoint Measurement: A New Type of Fundamental Measurement," J. Math. Psychol. 1, 1-27 (1964).

35. D. C. LuenBerger, Introduction to Linear and Nonlinear Programming, Addison-Wesley, Reading, Mass., 1973.

36. J. Marschak, "Rational Behavior, Uncertain Prospects, and Measurable Utility," Econometrica 18, 111-141 (1950).

37. D. MCFADDEN, "Conditional Logit Analysis of Qualitative Choice Behavior," in Frontiers in Econometrics, P. Zarembka (ed.), Academic Press, New York, 1970.

38. A. M. Mood ANd F. A. Greybill, Introduction to the Theory of Statistics, McGraw-Hill, New York, 1963.

39. S. H. NesLin, "Analyzing Consumer Response to Health Innovations: The Concept of Preference Inertia," Research Paper, Sloan School of Management, M.I.T., Cambridge, Mass., May 1976. 
40. S. H. Neslin, "Linking Product Features to Perceptions: Applications and Analysis of Graded Paired Comparisons," Proceedings of 1978 Educators' Conference, American Marketing Association, August 1978.

41. D. Pekelman and S. Sen, "Measurement and Estimation of Conjoint Utility Functions," J. Consumer Res. 5, 263-271 (1979).

42. E. A. Pessemier, Product Management: Strategy and Organization, WileyHamilton, New York, 1977.

43. J. Press, Applied Multivariate Analysis, Holt, Rinehart \& Winston, New York, 1971.

44. L. S. ShaPley, “Cardinal Utility from Intensity Comparisons,” RAND Report R-1683-PR, Santa Monica, Calif., July 1975.

45. S. M. Shugan, "The Cost of Thinking," Working Paper No. 7813, Graduate School of Management, University of Rochester, Rochester, N. Y., July 1978.

46. S. M. Shugan and V. Balachandran, "A Mathematical Programming Model for Optimal Product Line Structuring," Discussion Paper No. 265, Northwestern University, The Center for Mathematical Studies in Economics and Management Science, Evanston, Ill., April 1977.

47. S. M. Shugan and J. R. Hauser, "Designing and Building a Market Research Information System," Working Paper 602-001, Department of Marketing, Northwestern University, Evanston, Ill., revised February 1978.

48. A. Silk and G. L. URBan, "Pretest Market Evaluation of New Packaged Goods: A Model and Measurement Methodology," J. Marketing Res. 15, 171-191 (1978).

49. V. SRinivasan and A. Shocker, "Linear Programming Techniques for Multidimensional Analysis of Preferences," Psychometrika 38, 337-370 (1973).

50. S. S. Stevens, "Ratio Scales of Opinion," in Handbook of Measurement and Assessment in Behavioral Sciences, D. K. Whitla (ed.), Addison-Wesley, Reading, Mass., 1968.

51. H. M. TING, "Aggregation of Attributes for Multiattributed Utility Assessment," Technical Report No. 66, Operations Research Center, M.I.T., Cambridge, Mass. August 1971.

52. W. S. Torgerson, Theory and Methods of Scaling, John Wiley \& Sons, New York, 1958.

53. A. TVERsky, "A General Theory of Polynomial Conjoint Measurement," J. Math. Psychol. 4, 1-20, (1967).

54. G. L. URBan, "PERCEPTOR: A Model for Product Positioning," Mgmt. Sci. 8, 858-871 (1975).

55. J. von Neumann and O. Morgenstern, The Theory of Games and Economic Behavior, Ed. 2, Princeton University Press, Princeton, N. J., 1947.

56. S. S. Wilks, Mathematical Statistics, John Wiley \& Sons, New York, 1962.

57. Y. Wind and L. K. SPItz, "Analytical Approach to Marketing Decisions in Health-Care Organizations," Opns. Res. 24, 973-990 (1976). 